VOLUME 29 (2021) 15-49

DOI: $10.24330 /$ ieja. 851985

\title{
THE LOEWY SERIES OF AN FCP (DISTRIBUTIVE) RING EXTENSION
}

\author{
Gabriel Picavet and Martine Picavet-L'Hermitte \\ Received: 14 October 2019; Revised: 17 July 2020; Accepted: 19 July 2020 \\ Communicated by A. Çiğdem Özcan
}

\begin{abstract}
If $R \subseteq S$ is an extension of commutative rings, we consider the lattice $([R, S], \subseteq)$ of all the $R$-subalgebras of $S$. We assume that the poset $[R, S]$ is both Artinian and Noetherian; that is, $R \subseteq S$ is an FCP extension. The Loewy series of such lattices are studied. Most of main results are gotten in case these posets are distributive, which occurs for integrally closed extensions. In general, the situation is much more complicated. We give a discussion for finite field extensions.
\end{abstract}

Mathematics Subject Classification (2020): 13B02, 13B21, 13B22, 06E05 06D05, 13B30, 12F10

Keywords: FIP, FCP extension, minimal extension, support of a module, distributive lattice, Boolean lattice, atom, socle, Loewy series, Galois extension

\section{Introduction and notation}

If $L$ is a complete lattice, with smallest and greatest elements, its socle $\mathcal{S}(L)$ is defined as the supremum of all its atoms. Then the Loewy series of $L$ is defined by transfinite induction, where in particular $\mathcal{S}_{i+1}(L)=\mathcal{S}\left(\mathcal{S}_{i}(L)\right)$ for a positive integer $i$ (See Section 3 for more details). When $L$ is the lattice of submodules of a module, the Loewy series of $L$ is a well known topic and its theory is a long chapter of algebra, even when the base ring is non-commutative.

In this paper, we consider the category of commutative and unital rings, whose epimorphisms will be involved. If $R \subseteq S$ is a (ring) extension, we denote by [R,S] the set of all $R$-subalgebras of $S$ and set $] R, S[:=[R, S] \backslash\{R, S\}$ (with a similar definition for $[R, S[$ or $] R, S])$.

We will consider lattices of the following form. For an extension $R \subseteq S$, the poset $([R, S], \subseteq)$ is a complete lattice, where the supremum of any non void subset is the compositum of its elements, which we call product from now on and denote by $\Pi$ when necessary, and the infimum of any non void subset is the intersection of its elements. We emphasize on the following. If $R \subseteq S$ is a ring extension, our 
main interest is in the properties of the Loewy series related to the lattice $[R, S]$, and not in the lattice of $R$-submodules ( $R / C$-submodules) of $S / R$, where $C$ is the conductor of $R \subseteq S$, although there are some relations. Moreover, we only consider extensions of finite length, in a sense defined below, so that Loewy lengths are finite in this paper.

As a general rule, an extension $R \subseteq S$ is said to have some property of lattices if $[R, S]$ has this property. We use lattice definitions and properties described in [20].

The extension $R \subseteq S$ is said to have FIP (for the "finitely many intermediate algebras property") or is an FIP extension if $[R, S]$ is finite. A chain of $R$-subalgebras of $S$ is a set of elements of $[R, S]$ that are pairwise comparable with respect to inclusion. We will say that $R \subseteq S$ is chained if $[R, S]$ is a chain. We also say that the extension $R \subseteq S$ has FCP (or is an FCP extension) if each chain in $[R, S]$ is finite. Clearly, each extension that satisfies FIP must also satisfy FCP. A distributive FCP extension has FIP ([31, Theorem 4.28]). Dobbs and the authors characterized FCP and FIP extensions [7]. See also [16].

This paper is a continuation of our earlier paper [29], where we considered Boolean ring extensions. It is devoted to the study of Loewy series of an FCP (distributive) extension, a notion linked to Boolean extensions. As much as possible, we give results for FCP extensions that are not necessarily distributive, in particular, for the behavior of the Loewy series with respect to classical constructions of ring theory. It may be asked whether the distributivity property may be replaced with the modular condition, since the lattice of submodules of a module is evidently modular.

In a forthcoming paper, we study distributive extensions. Note that integrally closed FCP extensions are distributive (see Example 3.10 and [29, Proposition 2.4]).

Our main tool will be the minimal (ring) extensions, a concept that was introduced by Ferrand-Olivier [11]. In our context, minimal extensions coincide with atoms. They are completely known (see Section 2). Recall that an extension $R \subset S$ is called minimal if $[R, S]=\{R, S\}$. The key connection between the above ideas is that if $R \subseteq S$ has FCP, then any maximal (necessarily finite) chain $\mathcal{C}$ of $R$ subalgebras of $S, R=R_{0} \subset R_{1} \subset \cdots \subset R_{n-1} \subset R_{n}=S$, with length $\ell(\mathcal{C}):=$ $n<\infty$, results from juxtaposing $n$ minimal extensions $R_{i} \subset R_{i+1}, 0 \leq i \leq n-1$. An FCP extension is finitely generated, and (module) finite if integral. For any extension $R \subseteq S$, the length $\ell[R, S]$ of $[R, S]$ is the supremum of the lengths of chains of $R$-subalgebras of $S$. Notice that if $R \subseteq S$ has FCP, then there does exist some maximal chain of $R$-subalgebras of $S$ with length $\ell[R, S]$ [10, Theorem 4.11]. 
Any undefined material is explained at the end of the section or in the next sections.

Section 2 is devoted to some recalls and results on ring extensions and their lattice properties.

In Section 3, we study the Loewy series of an arbitrary FCP extension. As a first property, the Loewy series behaves well with respect to localization (Proposition 3.14). Let $R \subset S$ be a distributive FCP extension. Proposition 3.8 shows that the Loewy series $S_{0}:=R \subset \ldots \subset S_{i} \subset \ldots \subset S_{n+1}:=S$ is such that $S_{i} \subset S_{i+1}$ is a Boolean extension for each $i=0, \ldots, n-1$. In particular, Theorem 3.25 gives a characterization of such extensions verifying $[R, S]=\cup_{i=0}^{n}\left[S_{i}, S_{i+1}\right]$. We give computations of Loewy series for some special extensions or some subextensions, for example for Nagata extensions. We show also how to compute the Loewy series of some modules by using the Loewy series of a ring extension. We give many examples. For instance, if $R \subseteq S$ is an FCP almost-Prüfer extension with Prüfer

hull $\tilde{R}$, the Loewy series of $R \subseteq S$ is gotten by using the Loewy series of $R \subseteq \bar{R}$ and $R \subseteq \tilde{R}$ (Corollary 3.35). Note here that ring extensions whose Loewy length is 1 are, among others, Boolean extensions and pointwise minimal extensions (Corollary 3.9 and Proposition 3.12).

Section 4 specially deals with field extensions. We begin with the characterization of the Loewy series of a finite field extension $k \subseteq L$ by means of the Loewy series of $k \subseteq T$ and $k \subseteq U$, where $T$ (resp. $U$ ) is the separable (resp. radicial) closure of $k \subseteq L$ (Proposition 4.3). Loewy series of finite cyclic field extensions (they are necessarily distributive) are completely determined in Theorem 4.14.

We denote by $(R: S)$ the conductor of $R \subseteq S$. The integral closure of $R$ in $S$ is denoted by $\bar{R}^{S}$ (or by $\bar{R}$ if no confusion can occur). The characteristic of a field $k$ is denoted by $\mathrm{c}(k)$. A purely inseparable field extension is called radicial in this paper. In particular, if $k \subset L$ is a radicial FIP field extension, then $[k, L]$ is a chain.

Finally, $|X|$ is the cardinality of a set $X, \subset$ denotes proper inclusion and, for a positive integer $n$, we set $\mathbb{N}_{n}:=\{1, \ldots, n\}$.

\section{Recalls and results on ring extensions}

This section is devoted to two types of recalls: commutative rings and lattices.

2.1. Rings and ring extensions. A local ring is here what is called elsewhere a quasi-local ring. As usual, $\operatorname{Spec}(R)$ and $\operatorname{Max}(R)$ are the set of prime and maximal ideals of a ring $R$. The support of an $R$-module $E$ is $\operatorname{Supp}_{R}(E):=\{P \in \operatorname{Spec}(R) \mid$ $\left.E_{P} \neq 0\right\}$, and $\operatorname{MSupp}_{R}(E):=\operatorname{Supp}_{R}(E) \cap \operatorname{Max}(R)$. If $E$ is an $R$-module, $\mathrm{L}_{R}(E)$ (also denoted $L(E)$ ) is its length. 
If $R \subseteq S$ is a ring extension and $P \in \operatorname{Spec}(R)$, then $S_{P}$ is both the localization $S_{R \backslash P}$ as a ring and the localization at $P$ of the $R$-module $S$. We denote by $\kappa_{R}(P)$ the residual field $R_{P} / P R_{P}$ at $P$. An extension $R \subset S$ is called locally minimal if $R_{P} \subset S_{P}$ is minimal for each $P \in \operatorname{Supp}(S / R)$ or equivalently for each $P \in$ $\operatorname{MSupp}(S / R)$.

The following notions and results are deeply involved in the sequel.

Definition 2.1. [4, Definition 2.10] An extension $R \subset S$ is called $M$-crucial if $\operatorname{Supp}(S / R)=\{M\}$. Such $M$ is called the crucial (maximal) ideal $\mathcal{C}(R, S)$ of $R \subset S$.

Theorem 2.2. [11, Théorème 2.2] A minimal extension is crucial and is either integral ((module)-finite) or a flat epimorphism.

Recall that an extension $R \subseteq S$ is called Prüfer if $R \subseteq T$ is a flat epimorphism for each $T \in[R, S]$ (or equivalently, if $R \subseteq S$ is a normal pair) [17, Theorem 5.2]. In [27], we called an extension which is a minimal flat epimorphism, a Prüfer minimal extension. Three types of minimal integral extensions exist, characterized in the next theorem, (a consequence of the fundamental lemma of Ferrand-Olivier), so that there are four types of minimal extensions, mutually exclusive.

Theorem 2.3. [7, Theorem 2.2] Let $R \subset T$ be an extension and $M:=(R: T)$. Then $R \subset T$ is minimal and finite if and only if $M \in \operatorname{Max}(R)$ and one of the following three conditions holds:

(a) inert case: $M \in \operatorname{Max}(T)$ and $R / M \rightarrow T / M$ is a minimal field extension.

(b) decomposed case: There exist $M_{1}, M_{2} \in \operatorname{Max}(T)$ such that $M=M_{1} \cap$ $M_{2}$ and the natural maps $R / M \rightarrow T / M_{1}$ and $R / M \rightarrow T / M_{2}$ are both isomorphisms.

(c) ramified case: There exists $M^{\prime} \in \operatorname{Max}(T)$ such that $M^{\prime 2} \subseteq M \subset M^{\prime},[T / M$ : $R / M]=2$, and the natural map $R / M \rightarrow T / M^{\prime}$ is an isomorphism.

In each of the above cases, $M=\mathcal{C}(R, T)$.

2.2. Lattice properties. Let $R \subset S$ be an FCP extension, then $[R, S]$ is a complete Noetherian Artinian lattice, $R$ being the least element and $S$ the largest. In the context of the lattice $[R, S]$, some definitions and properties of lattices have the following formulations. (see [20])

An element $T \in[R, S]$ is called

(1) $\Pi$-irreducible (resp. $\cap$-irreducible) if $T=T_{1} T_{2}$ (resp. $T=T_{1} \cap T_{2}$ ) implies $T=T_{1}$ or $T=T_{2}$.

(2) an atom (resp. a co-atom) if and only if $R \subset T$ (resp. $T \subset S$ ) is a minimal extension. Therefore, an atom (resp. a co-atom) is $\Pi$-irreducible (resp. $\cap$-irreducible). 
We denote by $\mathcal{A}$ (resp. $\mathcal{C} \mathcal{A}$ ) the set of atoms (resp. co-atoms) of $[R, S]$. Theorems 2.2 and 2.3 show that there are four types of atoms.

(3) essential if $T \neq R$ and $U \cap T \neq R$ for each $U \in] R, S]$. If $N$ is an $R$ submodule of an $R$-module $M$, then $N$ is essential as a submodule if $N \cap N^{\prime} \neq \emptyset$ for any submodule $N^{\prime} \neq 0$ of $M$. Clearly, $T$ is essential if $T / R$ is an essential submodule of the $R$-module $S / R$.

(4) $R \subset S$ is called catenarian, or graded by some authors, if $R \subset S$ has FCP and all maximal chains between two comparable elements have the same length [30].

(5) $R \subseteq S$ is called distributive if intersection and product are each distributive with respect to the other. Actually, each distributivity implies the other [20, Exercise 5, page 33].

(6) Let $T \in[R, S]$. Then, $T^{\prime} \in[R, S]$ is called a complement of $T$ if $T \cap T^{\prime}=R$ and $T T^{\prime}=S$.

(7) An extension $R \subseteq S$ is called Boolean if $([R, S], \cap, \cdot)$ is a distributive lattice such that each $T \in[R, S]$ has a (necessarily unique) complement.

Proposition 2.4. [13, Theorem 1, p. 172] A distributive lattice of finite length is catenarian (the Jordan-Hölder chain condition holds).

Proposition 2.5. If $R \subseteq S$ has $F C P$, then a ring $T \in[R, S]$ is $\cap$-irreducible (resp. $\Pi$-irreducible) if and only if either $T=S$ (resp. $T=R$ ) or there is a unique $T^{\prime} \in[R, S]$ such that $T \subset T^{\prime}$ (resp. $T^{\prime} \subset T$ ) is minimal.

Proof. Obvious.

Definition 2.6. A ring extension $R \subseteq S$ is called arithmetic if $\left[R_{P}, S_{P}\right]$ is a chain for each $P \in \operatorname{Spec}(R)$.

An arithmetic extension is distributive by [25, Proposition 5.18].

In the next proposition, we need the following definition: A ring extension $R \subset S$ is called quadratic if each $t \in S$ is zero of a monic quadratic polynomial over $R$ ([15, definition page 430]).

Proposition 2.7. Let $R \subset S$ be a ring extension and let $T \in] R, S]$.

(1) If $T / R$ is an essential $R$-submodule of $S / R$, then $T$ is an essential $R$ subalgebra of $S$.

(2) If in addition, $R \subset S$ is quadratic, then $T$ is an essential $R$-subalgebra of $S$ if and only if $T / R$ is an essential $R$-submodule of $S / R$.

Proof. (1) Obvious. 
(2) One part is (1). Assume that $R \subset S$ is quadratic and that $T$ is an essential $R$-subalgebra of $S$. Let $N^{\prime}$ be a nonzero $R$-submodule of $S / R$. There exists an $R$-submodule $N$ of $S$ containing $R$ such that $N^{\prime}=N / R$. Let $t \in N \backslash R$. Then, $R \subset R[t]=R+R t \subseteq N$ because $t$ satisfies a monic quadratic polynomial over $R$. It follows that $R \neq T \cap R[t] \subseteq T \cap N$, which shows that $0 \neq(T / R) \cap(N / R)=$ $(T / R) \cap N^{\prime}$ and $T / R$ is an essential $R$-submodule of $S / R$.

Remark 2.8. There exist ring extensions such that the equivalence of (2) in Proposition 2.7 does not hold. Let $k \subset L$ be a radicial FIP field extension of degree $p^{2}$, where $\mathrm{c}(k)=p$. There exists a unique $K \in[k, L]$ such that $[K: k]=p$ because $[k, L]$ is a chain, so that $[k, L]=\{k, K, L\}$. Then, $K$ and $L$ are both essential $k$-subalgebras of $L$. But $K / k$ is not an essential $k$-vector subspace of $L / k$. Indeed, $[L: k]=p^{2}$ shows that there exists a basis $\left\{x_{1}, \ldots, x_{p^{2}}\right\}$ of the $k$-vector space $L$ such that $\left\{x_{1}, \ldots, x_{p}\right\}$ is a basis of the $k$-vector space $K$. Let $V:=k+k x_{p+1}$. Then, $(V / k) \cap(K / k)=0$ although $V / k \neq 0$.

\section{The Loewy series of an FCP (distributive) extension}

We first note that a distributive FCP extension $R \subset S$ has FIP ([31, Theorem $4.28])$. In this section, we associate a chain in $[R, S]$ to the lattice $[R, S]$, called the Loewy series of $[R, S]$.

Definition 3.1. [5, Definitions pages 47, 51 and 77], [20, page 29] Let $R \subset S$ be an FCP extension, $\mathcal{A}$ the set of atoms of $[R, S], \mathcal{C A}$ the set of co-atoms and $\mathcal{E}$ the set of essential elements.

(1) The socle of the extension $R \subset S$ is $\mathcal{S}[R, S]:=\prod_{A \in \mathcal{A}} A$.

(2) The radical of the extension $R \subset S$ is $\mathcal{R}[R, S]:=\cap_{A \in \mathcal{C} \mathcal{A}} A$.

(3) The Loewy series of the extension $R \subset S$ is the chain $\left\{S_{i}\right\}_{i=0}^{n}$ defined by induction as follows: $S_{0}:=R, S_{1}:=\mathcal{S}[R, S]$ and for each $i \geq 0$ such that $S_{i} \neq S$, we set $S_{i+1}:=\mathcal{S}\left[S_{i}, S\right]$.

(4) The Loewy length $£[R, S]$ of the extension $R \subset S$ is the least integer $n$ such that $S=S_{n}$. Of course, since $R \subset S$ has FCP, there is some integer $n$ such that $S_{n}=S_{n+1}=S$.

Proposition 3.2. Let $R \subset S$ be an FCP extension. Then, $£[R, S] \leq \ell[R, S] \leq$ $\mathrm{L}_{R}(S / R)$.

Proof. Since the Loewy series $\left\{S_{i}\right\}_{i=0}^{n}$ of $R \subset S$ is a chain, which is not necessarily maximal, we have $\ell[R, S] \geq \sum_{i=0}^{n-1} \ell\left[S_{i}, S_{i+1}\right] \geq n=£[R, S]$. Now, it exists $\left\{R_{j}\right\}_{j=0}^{m}$, a maximal chain such that $m=\ell[R, S][10$, Theorem 4.11]. Then, 
$\mathrm{L}_{R}(S / R)=\sum_{j=0}^{m-1} \mathrm{~L}_{R}\left(R_{j+1} / R_{j}\right)\left(\left[21\right.\right.$, Theorem 6, page 20]), with $\mathrm{L}_{R}\left(R_{j+1} / R_{j}\right) \geq$ 1 for each $j \in\{0, \ldots, m\}$, so that $\mathrm{L}_{R}(S / R) \geq \ell[R, S]$.

Proposition 3.3. Let $R \subset S$ be an FCP extension. Then, $\mathcal{S}[R, S]=\cap_{E \in \mathcal{E} E \text { and }}$ is the least element of $\mathcal{E}$.

Proof. By definition, $\mathcal{S}[R, S]:=\prod_{A \in \mathcal{A}} A$. Let $T \in \mathcal{E}$ and $A \in \mathcal{A}$. Then, $R \subset$ $T \cap A \subseteq A$ shows that $T \cap A=A$ since $R \subset A$ is minimal, which leads to $A \subseteq T$, so that any element of $\mathcal{A}$ is contained in any element of $\mathcal{E}$. In particular, $\mathcal{S}[R, S] \subseteq$ $\cap_{E \in \mathcal{E}} E(*)$. Let $\left.\left.U \in\right] R, S\right]$. Since $R \subset S$ has FCP, there exists some $A \in \mathcal{A}$ such that $A \subseteq U$, so that $A \cap U=A$. But $A \subseteq \mathcal{S}[R, S]$ shows that $R \subset A \subseteq U \cap \mathcal{S}[R, S]$. Then, $\mathcal{S}[R, S]$ is essential and $(*)$ gives the result.

We recall that an $R$-module $N \neq 0$ is called simple if it is an atom in the lattice $\Lambda(N)$ of $R$-submodules of $N$, which is equivalent to $N=R x$ for any nonzero $x \in N$ [20, Lemma 2.4.1]. In the following corollary, we set $\mathcal{M S}[R, S]:=\sum\{N \in \Lambda(S) \mid$ $R \subset N, N / R$ is simple in $\Lambda(S / R)\}$. (MS $[R, S]$ stands for module-socle). In view of [20, page 52], $\mathcal{M S}[R, S]$ is the intersection of the $R$-submodules $N$ of $S$ containing $R$ such that $N / R$ is essential in $S / R$.

Corollary 3.4. Let $R \subset S$ be an integral $M$-crucial FCP extension. Then $\mathcal{S}[R, S] \subseteq$ $\mathcal{M S}[R, S]$ with equality if $R \subset S$ is quadratic.

Proof. Set $k:=R / M$. Let $A \in \mathcal{A}$. Since $R \subset A$ is minimal, we have $M=(R$ : $A) \in \operatorname{Max}(R)$, so that $M(\mathcal{S}[R, S]) \subseteq R$. Let $x \in \mathcal{S}[R, S]$ and set $N:=R+R x \subseteq$ $R[x]$, which gives $M N \subseteq R$. Let $\bar{x}$ be the class of $x$ in $N / R$. Then, $N / R=R \bar{x}$. But $N / R$ is also a one-dimensional $k$-vector space generated by $\bar{x}$. It follows by $\left[21\right.$, Corollary 2, page 66] that $\mathrm{L}_{R}(N / R)=\mathrm{L}_{k}(N / R)=\operatorname{dim}_{k}(N / R)=1$ showing that $N / R$ is a simple $R$-submodule of $S / R$. Then, $x \in \mathcal{M S}[R, S]$, which leads to $\mathcal{S}[R, S] \subseteq \mathcal{M S}[R, S]$

Assume in addition that $R \subset S$ is quadratic. Let $x \in S$ be such that $N:=R+R x$ satisfies the following: $N / R$ is a simple $R$-submodule of $S / R$. Since $R \subset S$ is quadratic, it follows that $N \in[R, S]$. Moreover, the fact that $N / R$ is a simple $R$-submodule of $S / R$ shows that there is no $R$-submodule, and a fortiori, no $R$ subalgebra of $S$ strictly contained between $R$ and $N$, so that $N \in \mathcal{A} \subseteq \mathcal{S}[R, S]$. This property holding for any $R$-submodule $N$ of $S$ containing $R$ such that $N / R$ is a simple $R$-submodule, we get that $\mathcal{M S}[R, S] \subseteq \mathcal{S}[R, S]$, with equality because of the first part.

Remark 3.5. We use the example of Remark 2.8 to show that, in general, the two socles of Corollary 3.4 do not coincide. Let $k \subset L$ be a radicial FIP extension of 
degree $p^{2}$, where $\mathrm{c}(k)=p$. There exists a unique $K \in[k, L]$ such that $[K: k]=p$, so that $K$ is the unique atom of $[k, L]$, giving that $K=\mathcal{S}[k, L]$. But $[L: k]=p^{2}$ shows that there exists a basis $\left\{x_{1}:=1, \ldots, x_{p^{2}}\right\}$ of the $k$-vector space $L$, with $L=\sum_{i=1}^{p^{2}} k x_{i}$. For $i>1$, set $V_{i}:=k+k x_{i}$. Then, each $V_{i} / k$ is a simple subspace of $L / k$ and $L=\mathcal{M S}[k, L] \neq \mathcal{S}[k, L]=K$.

Lemma 3.6. Let $R \subset S$ be a distributive FCP extension (hence FIP) and let $S_{1}$ be its socle. Then, $S_{1}=\sup \{T \in[R, S] \mid R \subset T$ Boolean $\}$.

Proof. $R \subset S_{1}$ is Boolean because distributive and $S_{1}$ is a product of atoms of $R \subset S_{1}$ ([32, page 292]). Let $T \in[R, S]$ be such that $R \subset T$ is Boolean. It follows from [29, Theorem 3.1] that $T=\prod_{A \in \mathcal{B}} A$, where $\mathcal{B} \subseteq \mathcal{A}$, and then $T \subseteq S_{1}$.

Corollary 3.7. Let $R \subset S$ be a distributive FCP extension (hence FIP). Then $\mathcal{S}[R, T]=\mathcal{S}[R, S] \cap T$ holds for each $T \in] R, S]$.

Proof. Set $S_{1}:=\mathcal{S}[R, S], T_{1}:=\mathcal{S}[R, T]$ and $T_{1}^{\prime}:=S_{1} \cap T$. Because $T \neq R$, there exists some $A \in \mathcal{A}$ such that $R \subset A \subseteq T$. So, $R \subset A \subseteq S_{1} \cap T=T_{1}^{\prime} \subseteq S_{1}$. Since $R \subset S_{1}$ is Boolean, so is $R \subset T_{1}^{\prime}$ by [29, Proposition 3.12]. Then, $T_{1}^{\prime} \subseteq T_{1}$ in view of Lemma 3.6. But $R \subset T_{1}$ being also Boolean, it follows that $T_{1} \subseteq S_{1}$. From $T_{1}^{\prime} \subseteq T_{1} \subseteq T \cap S_{1}=T_{1}^{\prime}$ we infer that $T_{1}^{\prime}=T_{1}$.

The Loewy series of a distributive FCP extension provides a chain of Boolean subextensions of this extension.

Proposition 3.8. If $R \subset S$ is a distributive FCP extension (hence FIP) and $\left\{S_{i}\right\}_{i=0}^{n}$ is its Loewy series, then, $S_{i} \subset S_{i+1}$ is Boolean for each $0 \leq i \leq n-1$ whence, is either locally integral or locally Prüfer and $\ell[R, S]=\sum_{i=0}^{n-1} \ell\left[S_{i}, S_{i+1}\right]$.

Proof. By Definition 3.1 (3), for each $i \in\{0, \ldots, n-1\}, S_{i+1}:=\mathcal{S}\left[S_{i}, S\right]$ holds, so that $S_{i} \subset S_{i+1}$ is Boolean by Lemma 3.6. Since $R \subset S$ has FCP, the chain stops for some positive integer $n$ such that $S_{n}=S$. Deny, then there is some $S^{\prime}$ which is an atom of $\left[S_{n}, S\right]$, a contradiction.

For each $i \in\{0, \ldots, n-1\}, S_{i} \subset S_{i+1}$ is either locally integral or locally Prüfer [29, Proposition 3.5 and Corollary 3.20].

Since $R \subset S$ is distributive, $\ell[R, S]=\sum_{i=0}^{n-1} \ell\left[S_{i}, S_{i+1}\right]$ by Proposition 2.4.

Corollary 3.9. Let $R \subset S$ be a distributive FCP extension (hence FIP) and $\left\{S_{i}\right\}_{i=0}^{n}$ its Loewy series. Then,

(1) $£[R, S]=\ell[R, S]$ if and only if $[R, S]$ is a chain. In this case, $[R, S]=$ $\left\{S_{i}\right\}_{i=0}^{n}$. 
(2) $£[R, S]=1$ if and only if $R \subset S$ is Boolean.

Proof. Assume that $n=\ell[R, S]$. Proposition 3.8 implies $n=\ell[R, S] \Leftrightarrow \ell\left[S_{i}, S_{i+1}\right]=$ 1 for each $i \in\{0, \ldots, n-1\} \Leftrightarrow S_{i} \subset S_{i+1}$ is minimal for each $i \in\{0, \ldots, n-1\} \Leftrightarrow$ $S_{i} \subset S$ has only one atom (which is $S_{i+1}$ ) for each $i \in\{0, \ldots, n-1\}$. We show by induction on $i \in\{0, \ldots, n\}$ that if $T \in[R, S]$ is such that $\ell[R, T]=i$, then $T=S_{i}$. For $i=0$, obviously, $T=R=S_{0}$. Assume that the induction hypothesis holds for $i-1$; that is, $S_{i-1}$ is the only element $U$ of $[R, S]$ such that $\ell[R, U]=i-1$. Since $\ell[R, T]=i$ and $R \subset S$ is distributive and FCP, any element $T^{\prime}$ of $[R, S]$ such that $T^{\prime} \subset T$ is minimal satisfies $\ell\left[R, T^{\prime}\right]=i-1$, so that $T^{\prime}=S_{i-1}$ and $S_{i-1} \subset T$ is minimal, giving that $T$ is an atom of $\left[S_{i-1}, S\right]$, that is $T=S_{i}$. The induction holds for any $i \in\{0, \ldots, n\}$, and $[R, S]=\left\{S_{i}\right\}_{i=0}^{n}$ is a chain. The converse is immediate using the fact that $S_{i} \subset S$ has only one atom (which is $S_{i+1}$ ).

Again, by Proposition 3.8, $n=1 \Leftrightarrow S=S_{1} \Leftrightarrow R \subset S$ is Boolean.

Example 3.10. Let $R \subset S$ be an integrally closed FCP extension such that $R$ is a local ring. Then $[7$, Theorem 6.10$]$ says that $[R, S]$ is a chain, therefore distributive $\left[29\right.$, Proposition 2.3] and Corollary 3.9 shows that $[R, S]=\left\{S_{i}\right\}_{i=0}^{n}$.

Proposition 3.11. Let $R \subset S$ be an extension of length 2. The following conditions are equivalent:

(1) $[R, S]$ is a chain.

(2) $|[R, S]|=3$.

(3) $£[R, S]=2$.

If these conditions do not hold, then $£[R, S]=1$.

Proof. $(1) \Leftrightarrow(2)$ Obvious.

$(3) \Leftrightarrow(1)$ by Corollary 3.9.

Assume that these conditions do not hold, then $£[R, S]<\ell[R, S]$ by Proposition 3.2 leads to $£[R, S]=1$.

Recall that an extension $R \subset S$ is called pointwise minimal if $R \subset R[x]$ is minimal for each $x \in S \backslash R$. These extensions were studied by Cahen and the authors in [4].

Proposition 3.12. If $R \subset S$ is pointwise minimal, then, $£[R, S]=1$.

Proof. Any atom is a simple extension of $R$ (see [23, Page 370, before Lemma 1.2]). Conversely, let $x \in S \backslash R$, so that $R \subset R[x]$ is minimal and $R[x]$ is an atom of $[R, S]$. Then, $S=\prod_{x \in S \backslash R} R[x]$ gives that $S=\mathcal{S}[R, S]=S_{1}$ and $£[R, S]=1$.

In order to look at the behavior of Loewy series under localizations, we need the following Lemmata. 
Lemma 3.13. Let $R \subset S$ be an FCP extension, $T \subset U$ a subextension and $M \in$ $\operatorname{Supp}(S / R)$.

(1) If $T \subset U$ is minimal, then either $T_{M} \subset U_{M}$ is minimal, or $T_{M}=U_{M}$.

(2) If $T_{M} \subset U_{M}$ is minimal, there exists $V \in[T, S]$ such that $T \subset V$ is minimal with $V_{M}=U_{M}$.

Proof. (1) is [11, Lemme 1.3].

(2) If $T_{M} \subset U_{M}$ is minimal, let $Q:=\mathcal{C}\left(T_{M}, U_{M}\right) \in \operatorname{MSupp}_{T_{M}}\left(U_{M} / T_{M}\right) \subseteq$ $\operatorname{Max}\left(T_{M}\right)$. There exists $N \in \operatorname{Spec}(T)$, such that $Q=N_{M}$. In particular, we have $\left(T_{M}\right)_{Q} \subset\left(U_{M}\right)_{Q}$ minimal $(*)$ and $\left(T_{M}\right)_{P}=\left(U_{M}\right)_{P}$ for each $P \in \operatorname{Spec}\left(T_{M}\right), P \neq$ $Q(* *)$. Since $\left(T_{M}\right)_{Q}=\left(T_{M}\right)_{N_{M}}=T_{N}$ and $\left(U_{M}\right)_{Q}=\left(U_{M}\right)_{N_{M}}=U_{N}$ by $[2$, Proposition 7, page 85], (*) implies that $T_{N} \subset U_{N}$ is minimal. Then, there exists some $V \in[T, S]$ such that $T \subset V$ is minimal with $V_{N}=U_{N}=\left(U_{M}\right)_{Q}=\left(V_{M}\right)_{Q}[29$, Lemma 3.4]. It follows that $V_{N^{\prime}}=T_{N^{\prime}}$ for any $N^{\prime} \in \operatorname{Spec}(T), N^{\prime} \neq N$. But (**) gives, for $N^{\prime} \in \operatorname{Spec}(T)$ such that $P=N_{M}^{\prime}$ where $P \neq Q$, that $N^{\prime} \neq N$, so that $\left(T_{M}\right)_{P}=\left(U_{M}\right)_{P}=U_{N^{\prime}}=V_{N^{\prime}}=\left(V_{M}\right)_{P}$, which leads to $U_{M}=V_{M}$. To conclude, if $T_{M} \subset U_{M}$ is minimal, there exists $V \in[T, S]$ such that $T \subset V$ is minimal with $V_{M}=U_{M}$.

Proposition 3.14. Let $R \subset S$ be an FCP extension with Loewy series $\left\{S_{i}\right\}_{i=0}^{n}$. Then:

(1) Let $I$ be an ideal shared by $R$ and $S$. Then $\left\{S_{i} / I\right\}_{i=0}^{n}$ is the Loewy series of $R / I \subset S / I$.

(2) Let $M \in \operatorname{Supp}(S / R)$ and $\left\{S_{i}^{\prime}\right\}_{i=0}^{n_{M}}$ be the Loewy series of $\left[R_{M}, S_{M}\right]$. Then $S_{i}^{\prime}=\left(S_{i}\right)_{M}$ for each $i \in\left\{0, \ldots, n_{M}\right\}$, where $n_{M}=\inf \left\{i \in \mathbb{N}_{n} \mid M \notin\right.$ $\left.\operatorname{Supp}\left(S / S_{i}\right)\right\}$.

Proof. (1) Let $T, U \in[R, S]$. By [23, Corollary 1.4], $T \subset U$ is minimal if and only if $T / I \subset U / I$ is minimal. Then, an easy induction on $i$ shows that the Loewy series of $R / I \subset S / I$ is $\left\{S_{i} / I\right\}_{i=0}^{n}$.

(2) Let $M \in \operatorname{Supp}(S / R)$. We show by induction on $i \in\left\{0, \ldots, n_{M}\right\}$ that $S_{i}^{\prime}=$ $\left(S_{i}\right)_{M}$.

The induction hypothesis is satisfied for $i=0$.

Assume that it holds for $i \in\left\{0, \ldots, n_{M}-1\right\}$, that is $S_{i}^{\prime}=\left(S_{i}\right)_{M}$. Let $A$ be an atom of $\left[S_{i}, S\right]$, so that $S_{i} \subset A$ is minimal. Then, from Lemma 3.13, we infer that either $\left(S_{i}\right)_{M} \subset A_{M}$ is minimal, or $\left(S_{i}\right)_{M}=A_{M}$, so that $A_{M} \in\left[S_{i}^{\prime}, S_{i+1}^{\prime}\right]$. It follows that $\left(S_{i+1}\right)_{M} \subseteq S_{i+1}^{\prime}$.

Now $M \notin \operatorname{Supp}\left(S / S_{i}\right)$ leads to $S_{i}^{\prime}=\left(S_{i}\right)_{M}=S_{M}=S_{i+1}^{\prime}$ and $i \geq n_{M}$, a contradiction, so that $M \in \operatorname{Supp}\left(S / S_{i}\right)$. Let $B^{\prime}$ be an atom of $\left[\left(S_{i}\right)_{M}, S_{M}\right]$, so that 
$\left(S_{i}\right)_{M}=S_{i}^{\prime} \subset B^{\prime}$ is minimal. There exists some $U \in\left[S_{i}, S\right]$ such that $B^{\prime}=U_{M}$, with $\left(S_{i}\right)_{M} \subset U_{M}$ is minimal. By Lemma 3.13, there exists $B \in\left[S_{i}, S\right]$ such that $S_{i} \subset B$ is minimal, with $B_{M}=U_{M}=B^{\prime}$, so that $B \subseteq S_{i+1}$, giving $B^{\prime}=B_{M} \subseteq$ $\left(S_{i+1}\right)_{M}$. Since $S_{i+1}^{\prime}$ is the product of all atoms $B^{\prime}$ of $\left[\left(S_{i}\right)_{M}, S_{M}\right]$, we get that $S_{i+1}^{\prime} \subseteq\left(S_{i+1}\right)_{M}$. To conclude, $S_{i+1}^{\prime}=\left(S_{i+1}\right)_{M}$ and the induction hypothesis holds for $i+1$. As we have just seen before, we get $S_{i}^{\prime}=\left(S_{i}\right)_{M}=S_{M}=S_{i+1}^{\prime}$ as soon as $M \notin \operatorname{Supp}\left(S / S_{i}\right)$, so that $n_{M}=\inf \left\{i \in \mathbb{N}_{n} \mid M \notin \operatorname{Supp}\left(S / S_{i}\right)\right\}$.

Corollary 3.15. Let $R \subset S$ be an FCP extension. Then,

$$
£[R, S]=\max \left\{£\left[R_{M}, S_{M}\right] \mid M \in \operatorname{MSupp}(S / R)\right\} .
$$

Proof. Let $M \in \operatorname{Supp}(S / R)$ and $\left\{S_{i}^{\prime}\right\}_{i=0}^{n_{M}}$ be the Loewy series of $\left[R_{M}, S_{M}\right]$. Set $n:=£[R, S]$. We proved in Proposition 3.14 that $S_{i}^{\prime}=\left(S_{i}\right)_{M}$ for each $i \in$ $\left\{0, \ldots, n_{M}\right\}$, where $n_{M}=£\left[R_{M}, S_{M}\right]=\inf \left\{i \in \mathbb{N}_{n} \mid M \notin \operatorname{Supp}\left(S / S_{i}\right)\right\}$. Then, for any $M \in \operatorname{Supp}(S / R), £\left[R_{M}, S_{M}\right] \leq £[R, S]$. Assume that for any $M \in$ $\operatorname{Supp}(S / R), £\left[R_{M}, S_{M}\right]<£[R, S]=n$. It follows that $S_{n-1}^{\prime}=\left(S_{n-1}\right)_{M}=$ $S_{M}$, and hence $S_{n-1}=S$, contradicting the definition of $n$. Then, $£[R, S]=$ $\max \left\{£\left[R_{M}, S_{M}\right] \mid M \in \operatorname{MSupp}(S / R)\right\}$.

Example 3.16. We use the example of Remark 2.8 in order to exhibit a computation of $n_{M}$ in Proposition 3.14:

$k \subset L$ is a radicial field extension of degree $p^{2}$ and $K$ is the only proper subalgebra of $L$. Set $R:=k^{2}, R_{1}:=\left[k[X] /\left(X^{2}\right)\right] \times k, R_{2}:=k \times K, R_{3}:=k \times L, S:=$ $\left[k[X] /\left(X^{2}\right)\right] \times L, M:=0 \times k$ and $N:=k \times 0$. Then, $\operatorname{Max}(R)=\{M, N\}$ with $M \neq N$. Moreover, $k \subset k[X] /\left(X^{2}\right)$ is a minimal ramified extension and $S=R_{1} R_{3}$. By [6, Lemma III.3 (d)], $[R, S]=\left\{R, R_{1}, R_{2}, R_{1} R_{2}, R_{3}, S\right\}$. We have the following diagram, where $R_{1} R_{2}=\left[k[X] /\left(X^{2}\right)\right] \times K$ :

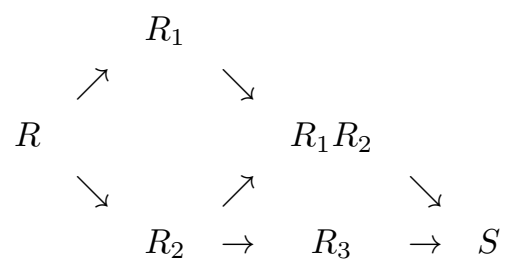

From [6, Proposition III.4], we deduce that $R \subset R_{1}$ is a minimal extension with $\mathcal{C}\left(R, R_{1}\right)=M, R \subset R_{2}$ is a minimal extension with $\mathcal{C}\left(R, R_{2}\right)=N \neq M$, so that $T$ is an atom of $[R, S]$ if and only if $T \in\left\{k \times K,\left[k[X] /\left(X^{2}\right)\right] \times k\right\}=\left\{R_{1}, R_{2}\right\}$, and then $S_{1}=R_{1} R_{2}$. Using again [6, Proposition III.4], we get that $S_{1} \subset S$ is minimal, so that $S_{2}=S$. Then, $n=2$. Now, $\left(S_{1}\right)_{M}=\left(R_{1}\right)_{M}=S_{M}=\left(S_{2}\right)_{M}$, so that $n_{M}=1$ and $\left(S_{1}\right)_{N}=\left(R_{2}\right)_{N} \neq S_{N}=\left(R_{3}\right)_{N}=\left(S_{2}\right)_{N}$, whence $n_{N}=2$. 
Let $R \subset S$ be an FCP extension and $\operatorname{MSupp}(S / R):=\left\{M_{1}, \ldots, M_{n}\right\}$. Consider the map $\varphi:[R, S] \rightarrow \prod_{i=1}^{n}\left[R_{M_{i}}, S_{M_{i}}\right]$ defined by $\varphi(T):=\left(T_{M_{1}}, \ldots, T_{M_{n}}\right)$. Then $\varphi$ is injective [7, Theorem 3.6]. In [29], we called $R \subseteq S$ a $\mathcal{B}$-extension if $\varphi$ is bijective ( $\mathcal{B}$ stands for bijective). We proved in [29, Proposition 2.21] that $R \subseteq S$ is a $\mathcal{B}$-extension if and only if $R / P$ is local for each $P \in \operatorname{Supp}(S / R)$. This condition holds in case $\operatorname{Supp}(S / R) \subseteq \operatorname{Max}(R)$, and, in particular, if $R \subset S$ is integral. The Loewy series of such extensions have nice properties.

Proposition 3.17. Let $R \subset S$ be an $F C P \mathcal{B}$-extension. For each $M \in \operatorname{MSupp}(S / R)$, set $\mathcal{A}_{M}:=\{A \in \mathcal{A} \mid \mathcal{C}(R, A)=M\}$. Then:

(1) $\mathcal{S}\left[R_{M}, S_{M}\right]=\prod_{A \in \mathcal{A}_{M}} A_{M}$

(2) There exists a (unique) $S_{1, M} \in[R, S]$ such that $\left(S_{1, M}\right)_{M}=\mathcal{S}\left[R_{M}, S_{M}\right]$ and $\left(S_{1, M}\right)_{M^{\prime}}=R_{M^{\prime}}$ for any $M^{\prime} \in \operatorname{Spec}(R), M^{\prime} \neq M$. In particular, $S_{1, M}=\prod_{A \in \mathcal{A}_{M}} A$.

(3) $\mathcal{A}=\cup_{M \in \operatorname{MSupp}(S / R)} \mathcal{A}_{M}$ and $S_{1}=\prod_{M \in \operatorname{MSupp}(S / R)} S_{1, M}$.

Proof. (1) $(\mathcal{S}[R, S])_{M}=\mathcal{S}\left[R_{M}, S_{M}\right]$ by Proposition 3.14. It follows $\mathcal{S}\left[R_{M}, S_{M}\right]=$ $\prod_{A \in \mathcal{A}} A_{M}$. Let $M^{\prime} \in \operatorname{MSupp}(S / R), M^{\prime} \neq M$ and $A^{\prime} \in \mathcal{A}_{M^{\prime}}$. Then, $\mathcal{C}\left(R, A^{\prime}\right)=M^{\prime}$ implies $A_{M}^{\prime}=R_{M}$, so that $\mathcal{S}\left[R_{M}, S_{M}\right]=\prod_{A \in \mathcal{A}_{M}} A_{M}$.

(2) Set $\operatorname{MSupp}(S / R)=\left\{M_{1}, \ldots, M_{n}\right\}$. Since $R \subset S$ is a $\mathcal{B}$-extension, the bijective map $\varphi:[R, S] \rightarrow \prod_{i=1}^{n}\left[R_{M_{i}}, S_{M_{i}}\right]$ shows that there exists a unique $S_{1, M} \in$ $[R, S]$ such that $\left(S_{1, M}\right)_{M}=\mathcal{S}\left[R_{M}, S_{M}\right]=\prod_{A \in \mathcal{A}_{M}} A_{M}$ and $\left(S_{1, M}\right)_{M^{\prime}}=R_{M^{\prime}}$ for any $M^{\prime} \in \operatorname{Spec}(R), M^{\prime} \neq M$. Moreover, for $A \in \mathcal{A}_{M}$, we have $A_{M^{\prime}}=R_{M^{\prime}}$, giving $\prod_{A \in \mathcal{A}_{M}} A_{M^{\prime}}=R_{M^{\prime}}$, so that $S_{1, M}=\prod_{A \in \mathcal{A}_{M}} A$.

(3) $\mathcal{A}=\cup_{M \in \operatorname{MSupp}(S / R)} \mathcal{A}_{M}$ because, for each $A \in \mathcal{A}$, we have $\mathcal{C}(R, A) \in$ $\operatorname{MSupp}(S / R)$, which leads to $S_{1}=\prod_{A \in \mathcal{A}} A=\prod_{M \in \operatorname{MSupp}(S / R)}\left(\prod_{A \in \mathcal{A}_{M}} A\right)$

$=\prod_{M \in \operatorname{MSupp}(S / R)} S_{1, M}$.

Proposition 3.18. Let $R \subset S$ be an integral arithmetic FCP extension with Loewy series $\left\{S_{i}\right\}_{i=0}^{n}$. For each $M \in \operatorname{MSupp}(S / R)$, let $\left\{R_{M, i}\right\}_{i=0}^{n_{M}}$ be the maximal chain of $\left[R_{M}, S_{M}\right]$, set

$$
\mathcal{A}_{M}:=\{A \in \mathcal{A} \mid \mathcal{C}(R, A)=M\} \text { and } m:=\sup _{M \in \operatorname{MSupp}(S / R)}\left(£\left[R_{M}, S_{M}\right]\right) .
$$

Then:

(1) For each $M \in \operatorname{MSupp}(S / R),\left|\mathcal{A}_{M}\right|=1$. Let $T_{1, M}$ be the unique element of $\mathcal{A}_{M}$

(2) $\mathcal{S}[R, S]=\prod_{M \in \operatorname{MSupp}(S / R)} T_{1, M}$.

(3) For each $i \in \mathbb{N}_{m}$, the atoms of $\left[S_{i-1}, S\right]$ are the $T_{i, M}$ such that, for each $M, M^{\prime} \in \operatorname{MSupp}(S / R), M^{\prime} \neq M,\left(T_{i, M}\right)_{M}=R_{M, i}$ if $i \leq n_{M}$ and $\left(T_{i, M}\right)_{M}$ 
$=S_{M}$ if $i>n_{M}$, with $\left(T_{i, M}\right)_{M^{\prime}}=R_{M^{\prime}, i-1}$. Also, $S_{i}=\prod_{M \in \operatorname{MSupp}(S / R)} T_{i, M}$. In particular, $£[R, S]=m$.

(4) $\ell[R, S]=\sum_{i=0}^{n-1} \ell\left[S_{i}, S_{i+1}\right]=\sum_{M \in \operatorname{MSupp}(S / R)} £\left[R_{M}, S_{M}\right]$.

Proof. Since $R \subset S$ is arithmetic, for each $M \in \operatorname{MSupp}(S / R),\left\{R_{M, i}\right\}_{i=0}^{n_{M}}$ is the Loewy series of $R_{M} \subset S_{M}$ by Corollary 3.9.

(1) Let $M \in \operatorname{MSupp}(S / R)$. Since $R_{M} \subset S_{M}$ is a chain, $R_{M, 1}$ is its only atom. From Lemma 3.13, we deduce that there is $T_{1, M} \in[R, S]$ such that $R \subset T_{1, M}$ is minimal with $\left(T_{1, M}\right)_{M}=R_{M, 1}$ and $\left(T_{1, M}\right)_{M^{\prime}}=R_{M^{\prime}}$ for $M^{\prime} \in \operatorname{MSupp}(S / R), M^{\prime} \neq$ $M(*)$. Since $R \subset S$ is integral, it is a $\mathcal{B}$-extension by [29, Proposition 2.21], so that $T_{1, M} \in \mathcal{A}_{M}$ is the only element of $\mathcal{A}$ satisfying (*). Then, $\mathcal{A}_{M}=\left\{T_{1, M}\right\}$ and $\left|\mathcal{A}_{M}\right|=1$.

(2) We get that $\mathcal{S}[R, S]=\prod_{M \in \operatorname{MSupp}(S / R)}\left(\prod_{A \in \mathcal{A}_{M}} A\right)=\prod_{M \in \operatorname{MSupp}(S / R)} T_{1, M}$ by Proposition 3.17.

(3) We proved in Proposition 3.14 that for $M \in \operatorname{Supp}(S / R)$ and $\left\{S_{i}^{\prime}\right\}_{i=0}^{n_{M}}$ the Loewy series of $\left[R_{M}, S_{M}\right]$, then $S_{i}^{\prime}=\left(S_{i}\right)_{M}$ for each $i \in\left\{0, \ldots, n_{M}\right\}$, where $n_{M}=$ $\inf \left\{i \in \mathbb{N}_{n} \mid M \notin \operatorname{Supp}\left(S / S_{i}\right)\right\}$. In particular, $S_{i}^{\prime}=\left(S_{i}\right)_{M}=R_{M, i}$. Let $T$ be an atom of $\left[S_{i-1}, S\right]$, and set $M:=\mathcal{C}\left(S_{i-1}, T\right) \cap R \in \operatorname{MSupp}_{R}\left(S / S_{i-1}\right)$, so that $i \leq n_{M}$ and $S_{i-1}^{\prime} \subset T_{M}$ is minimal. Moreover, by minimality of $S_{i-1} \subset T$, we get $T_{M^{\prime}}=\left(S_{i-1}\right)_{M^{\prime}}$ for $M^{\prime} \in \operatorname{MSupp}(S / R), M^{\prime} \neq M$. Since, $R \subset S$ is a $\mathcal{B}$-extension, it follows that $T$ is unique for a given $M$, and of the form $T_{i, M}$ such that, for each $M, M^{\prime} \in \operatorname{MSupp}(S / R), M^{\prime} \neq M,\left(T_{i, M}\right)_{M}=R_{M, i}$ if $i \leq n_{M}$ and $\left(T_{i, M}\right)_{M}=S_{M}$ if $i>n_{M}$, and $\left(T_{i, M}\right)_{M^{\prime}}=R_{M^{\prime}}$. Moreover, $S_{i}=\prod_{M \in \operatorname{MSupp}(S / R)} T_{i, M}$. In particular, $£[R, S]=\sup _{M \in \operatorname{MSupp}(S / R)}\left(£\left[R_{M}, S_{M}\right]\right)=m$ by Corollary 3.15 .

(4) Since $R \subset S$ is arithmetic, it is distributive (Definition 2.6), so that $\ell[R, S]=$ $\sum_{i=0}^{n-1} \ell\left[S_{i}, S_{i+1}\right]$ by Proposition 3.8. But, $S_{i} \subset S_{i+1}$ is Boolean, so that $\ell\left[S_{i}, S_{i+1}\right]$ is the number of atoms of $S_{i} \subset S_{i+1}$ by [29, Theorem 3.2]. In view of (3), they are gotten, for each $S_{i} \subset S_{i+1}$, by the elements of the chain $\left[R_{M}, S_{M}\right]$ which are of the form $R_{M, i+1}$, that is for $i<n_{M}$. Then, $\ell\left[S_{i}, S_{i+1}\right]=\left|\left\{R_{M, i+1} \mid i<n_{M}\right\}\right|$, giving $\ell[R, S]=\sum_{M \in \operatorname{MSupp}(S / R)}\left(n_{M}-1\right)$.

In Corollary 3.9, we proved that if $[R, S]$ is a chain, then $[R, S]=\left\{S_{i}\right\}_{i=0}^{n}$. We introduce the following property:

Definition 3.19. An FCP extension $R \subset S$ with Loewy series $\left\{S_{i}\right\}_{i=0}^{n}$ is said to satisfy the property $(\mathcal{P})$ (or is a $\mathcal{P}$-extension) if $[R, S]=\cup_{i=0}^{n-1}\left[S_{i}, S_{i+1}\right]$. (The Loewy series gives a partition of $[R, S]$.)

Here is an example of such a $\mathcal{P}$-extension. 
Example 3.20. In [28, Example 5.17 (2)], we gave the following example: Set $k:=\mathbb{Q}, L:=k[x]$, where $x:=\sqrt{3}+\sqrt{2}$ and $k_{i}:=k[\sqrt{i}], i=2,3,6$. Then, $[k, L]=\left\{k, k_{2}, k_{3}, k_{6}, L\right\}$ and the following diagram holds:

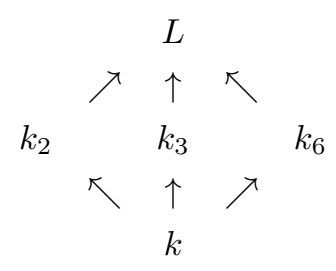

so that $k \subset L$ is a non distributive extension of length 2 (because a diamond [31, Theorem 4.7]), with atoms $k_{i}, i=2,3,6$ so that $L=k_{i} k_{j}$, for any $i, j \in$ $\{2,3,6\}, i \neq j$. Then, $\mathcal{S}[k, L]=L=S_{1},[k, L]=\left[k, S_{1}\right]$ and $k \subset L$ is a $\mathcal{P}$ extension.

Although many properties of $\mathcal{P}$-extensions will be gotten for a distributive extension, we begin to give two results for a non necessarily distributive $\mathcal{P}$-extension.

Proposition 3.21. Let $R \subset S$ be an FIP extension with Loewy series $\left\{S_{i}\right\}_{i=0}^{n}$. Then, $|[R, S]| \geq \sum_{i=0}^{n-1}\left|\left[S_{i}, S_{i+1}\right]\right|+1-n$, with equality if and only if $R \subset S$ is a $\mathcal{P}$-extension.

Proof. Since $\cup_{i=0}^{n-1}\left[S_{i}, S_{i+1}\right] \subseteq[R, S]$, we get that $\left|\cup_{i=0}^{n-1}\left[S_{i}, S_{i+1}\right]\right| \leq|[R, S]|$. But $\left\{S_{i}\right\}_{i=0}^{n}$ is an ascending chain, so that $\left[S_{i-1}, S_{i}\right] \cap\left[S_{i}, S_{i+1}\right]=\left\{S_{i}\right\}$ for each $i \in \mathbb{N}_{n-1}$ and $\left[S_{j}, S_{j+1}\right] \cap\left[S_{i}, S_{i+1}\right]=\emptyset$ for any $(i, j)$ such that $j \neq i, i-1, i+1$. Then, $\left|\cup_{i=0}^{n-1}\left[S_{i}, S_{i+1}\right]\right|=\sum_{i=0}^{n-1}\left|\left[S_{i}, S_{i+1}\right]\right|+1-n$, since there are $n-1$ elements $S_{i}$ common to two distinct subsets $\left[S_{j}, S_{j+1}\right]$. The equality holds if and only if $\cup_{i=0}^{n-1}\left[S_{i}, S_{i+1}\right]=[R, S]$ if and only if $R \subset S$ satisfies property $(\mathcal{P})$.

Theorem 3.22. Let $R \subset S$ be an FCP $\mathcal{P}$-extension with Loewy series $\left\{S_{i}\right\}_{i=0}^{n}$. Then $R \subset S$ is distributive if and only if $S_{i} \subset S_{i+1}$ is Boolean for each $0 \leq i \leq n-1$. If these conditions hold, then $R \subset S$ has FIP.

Proof. One part of the proof is Proposition 3.8.

Conversely, assume that $R \subset S$ satisfies property $(\mathcal{P})$ and that $S_{i} \subset S_{i+1}$ is Boolean for each $i \leq n$. Let $T, U, V \in[R, S]$ be such that $U T=V T$ and $U \cap T=$ $V \cap T$. We claim that $U=V$. This will prove that $R \subset S$ is distributive by [5, Theorem 1.6, page 9]. The result is obvious if $S \in\{U, V, T\}$. Then, choose $i, j, k \in\{0, \ldots, n-1\}$ such that $T \in\left[S_{i}, S_{i+1}\left[, U \in\left[S_{j}, S_{j+1}\left[, V \in\left[S_{k}, S_{k+1}[\right.\right.\right.\right.\right.$. Set $l:=\sup (j+1, k+1)$ and $l^{\prime}:=\inf (j, k)$. This yields that $l^{\prime}<l$ and $S_{l^{\prime}} \subseteq U, V \subset S_{l}$.

Consider the different cases: 
(1) If $i=j=k$, then $U=V$, because $T, U, V \in\left[S_{i}, S_{i+1}\right]$, which is Boolean, and then distributive.

(2) Assume $i \geq l$, so that $U, V \subset S_{l} \subseteq S_{i} \subseteq T$. Then, $U=U \cap T=V \cap T=V$.

(3) Assume that $i<l^{\prime}$, so that $i+1 \leq l^{\prime}$ which implies $T \subset S_{i+1} \subseteq S_{l^{\prime}} \subseteq U, V$. Then, $U=U T=V T=V$.

(4) The last case to consider (which has two subcases) is when $l^{\prime} \leq i<l(*)$. There is no harm to assume $j \leq k$. In this case, $l^{\prime}=j$ and $l=k+1$, so that $(*)$ yields $j \leq i \leq k$.

If $j=i \leq k$, we can take $i<k$ because of (1), and then $i+1 \leq k$. It follows that $U, T \subset S_{i+1} \subseteq S_{k} \subseteq V$. Then, $V=V T=U T$ and $T=T \cap V=T \cap U$ leads to $T \subseteq U$, whence $V=U T=U$.

If $j<i \leq k$, then, by $j+1 \leq i$, we obtain $U \subset S_{j+1} \subseteq S_{i} \subseteq T$ which gives $U \cap T=U=V \cap T \subset S_{i} \subseteq S_{k} \subseteq V$, so that $S_{i} \subseteq T \cap V=U$, a contradiction which shows that this case does not occur.

To conclude, $U=V$ in each case and $R \subset S$ is distributive.

The last result holds since an FCP distributive extension has FIP.

Corollary 3.23. Let $R \subset S$ be a distributive FCP (hence FIP) $\mathcal{P}$-extension, with Loewy series $\left\{S_{i}\right\}_{i=0}^{n}$ and $\left.T \in\right] R, S[$. There is some $k \in\{0, \ldots, n-1\}$, such that $T \in$ $\left[S_{k}, S_{k+1}\left[\right.\right.$ and then $\left\{S_{i}\right\}_{i=0}^{k} \cup\{T\}$ is the Loewy series of $R \subset T$ and $\{T\} \cup\left\{S_{i}\right\}_{i=k+1}^{n}$ is the Loewy series of $T \subset S$. Moreover, $R \subset T$ and $T \subset S$ are $\mathcal{P}$-extensions.

Proof. Since $R \subset S$ is a $\mathcal{P}$-extension, there is some $k \in\{0, \ldots, n-1\}$ such that $T \in\left[S_{k}, S_{k+1}\left[\right.\right.$, so that $S_{k} \subseteq T \subset S_{k+1}$. Let $\left\{T_{i}\right\}_{i=0}^{m}$ (resp. $\left\{T_{i}^{\prime}\right\}_{i=0}^{r}$ ) be the Loewy series of $R \subset T$ (resp. $T \subset S$ ). By definition of the socle of an extension, we have obviously $S_{i}=T_{i}$ for each $i \in\{0, \ldots, k\}$. Moreover, $S_{k} \subset S_{k+1}$ is Boolean by Proposition 3.8, which implies that $T$ is a product of atoms of $S_{k} \subset S[29$, Theorem 3.2]. In fact, $T$ is a product of the atoms of $S_{k} \subset S$ contained in $T$, so that $T$ is the product of atoms of $S_{k} \subset T$, giving $T=\mathcal{S}\left[S_{k}, T\right]$. Then, $T_{k+1}=T$ and $m=k+1$. Now, $T=T_{0}^{\prime}$. From [29, Proposition 3.12], we deduce that $T \subset S_{k+1}$ is also Boolean, and $S_{k+1}$ is the product of the atoms of $\left[T, S_{k+1}\right]$. We claim that $S_{k+1}=\mathcal{S}[T, S]$. Deny, so that there exists some atom $A$ of $[T, S]$ which is not in $\left[T, S_{k+1}\right]$. But $T \subset A$ is a minimal extension. Since $R \subset S$ is a $\mathcal{P}$-extension, we get that $A \in \cup_{i=k+1}^{n-1}\left[S_{i}, S_{i+1}\right]$ and then $T \subset S_{k+1} \subset A$, a contradiction. Then, $S_{k+1}=T_{1}^{\prime}$ and the other terms of the Loewy series of $T \subset S$ are the $S_{i}$ for $i \in\{k+2, \ldots, n\}$. The last property follows easily.

In the next result, we use the radical $\mathcal{R}$ of an extension (Definition $3.1(2)$ ). 
Corollary 3.24. Let $R \subset S$ be a distributive FCP $\mathcal{P}$-extension (hence FIP), with Loewy series $\left\{S_{i}\right\}_{i=0}^{n}$. Then $S_{i}=\mathcal{R}\left[S_{i}, S_{i+1}\right]$ for each $i \in\{0, \ldots, n-1\}$ and $S_{n-1}=\mathcal{R}[R, S]$.

Proof. In view of Theorem 3.22, $S_{i} \subset S_{i+1}$ is Boolean for each $i \in\{0, \ldots, n-1\}$. Then $S_{i}=\mathcal{R}\left[S_{i}, S_{i+1}\right]$ for each $i \leq n-1$ by de Morgan's law [31, Theorems 3.43 and 5.1] and the equivalences of [32, page 292]. Indeed, since $S_{i} \subset S_{i+1}$ is Boolean, $S_{i}$ is the complement of $S_{i+1}$. Now, $S_{i+1}$ is the product of atoms of $\left[S_{i}, S_{i+1}\right]$, which implies that $S_{i}$ is the intersection of co-atoms of $\left[S_{i}, S_{i+1}\right]$; that is, $\mathcal{R}\left[S_{i}, S_{i+1}\right]$.

Since $R \subset S$ is a $\mathcal{P}$-extension, $[R, S]=\cup_{i=0}^{n-1}\left[S_{i}, S_{i+1}\right]$. Let $A \in \mathcal{C} \mathcal{A}$. There is some $i \in\{0, \ldots, n-1\}$ with $A \in\left[S_{i}, S_{i+1}\right]$, whence $A$ is comparable to any $S_{i}$. But $A \subset S$ is minimal, so that $S_{n-1} \subseteq A$ yields that $A$ is a co-atom of $\left[S_{n-1}, S\right]$. It follows that $S_{n-1} \subseteq \cap_{A \in \mathcal{C} \mathcal{A}} A=\mathcal{R}[R, S] \subseteq \mathcal{R}\left[S_{n-1}, S\right]=S_{n-1}$ by the first part of the proof.

Proposition 2.5 says that when $R \subseteq S$ has FCP, $T \in[R, S]$ is $\Pi$-irreducible if and only if either $T=R$ or there is a unique $T^{\prime} \in[R, S]$ such that $T^{\prime} \subset T$ is minimal. For an FCP distributive $\mathcal{P}$-extension, these elements can be characterized thanks to the Loewy series. In fact, the following theorem characterizes FCP distributive $\mathcal{P}$-extensions.

Theorem 3.25. Let $R \subset S$ be a distributive FCP (hence FIP) extension with Loewy series $\left\{S_{i}\right\}_{i=0}^{n}$. Then, $R \subset S$ is a $\mathcal{P}$-extension if and only if the following condition holds: any $T \in] R, S]$ is $\Pi$-irreducible in $[R, S]$ if and only if there exists some $i \leq n-1$ such that $T$ is an atom of $\left[S_{i}, S_{i+1}\right]$.

Proof. Assume first: $R \subset S$ is a $\mathcal{P}$-extension. Let $T \in] R, S]$ be $\Pi$-irreducible in $[R, S]$. There is some $i \in\{0, \ldots, n-1\}$ such that $T \in\left[S_{i}, S_{i+1}\right]$, which is Boolean. It follows that $T$ is a product of $m$ atoms of $\left[S_{i}, S_{i+1}\right]$ by [29, Theorem 3.2]. But, $T$ being $\Pi$-irreducible in $[R, S]$ is a fortiori $\Pi$-irreducible in $\left[S_{i}, S_{i+1}\right]$, giving $m=1$, so that $T$ is itself an atom of $\left[S_{i}, S_{i+1}\right]$. Conversely, let $T$ be an atom of some $\left[S_{i}, S_{i+1}\right]$. We show that $T$ is $\Pi$-irreducible in $[R, S]$. Deny, and let $U, V \in[R, S] \backslash\{T\}$ be such that $T=U V$, so that $U, V \subset T \subseteq S_{i+1}(*)$. Because of property $(\mathcal{P})$, there exist $j, k \in\{0, \ldots, n-1\}$ such that $U \in\left[S_{j}, S_{j+1}\left[\right.\right.$ and $V \in\left[S_{k}, S_{k+1}\right.$ [. Moreover, (*) implies that $j, k \leq i$. We cannot have $j=k=i$ since $T$ is an atom of $\left[S_{i}, S_{i+1}\right]$. Assume that only one $U, V$ is in $\left[S_{i}, S_{i+1}\right]$, for instance, $U \in\left[S_{i}, S_{i+1}\right]$, so that $i=j$. Then, $V \subset S_{i} \subseteq U \subset T$ leads to $U V=U \subset T$, a contradiction. For the remaining case $j, k<i$, we have $j+1, k+1 \leq i$ and $U, V \notin\left[S_{i}, S_{i+1}\right]$. We get that $U V \subseteq S_{i} \subset T$, again a contradiction. Then, $T$ is $\Pi$-irreducible. 
Now, we show that $[R, S]=\cup_{i=0}^{n-1}\left[S_{i}, S_{i+1}\right]$ if the following condition holds: any $T \in] R, S]$ is $\Pi$-irreducible if and only if there exists some $i \in\{0, \ldots, n-1\}$ such that $T$ is an atom of $\left[S_{i}, S_{i+1}\right]$. Let $\left.\left.U \in\right] R, S\right]$, so that $U=\prod_{j=1}^{m} T_{j}$, for some positive integer $m$, where $T_{j}$ is $\Pi$-irreducible for each $j$ by [29, Proposition $2.9]$ and $T_{j} \neq R$. The hypothesis gives that for each $j$, there exists a unique $i_{j} \in\{0, \ldots, n-1\}$ such that $T_{j}$ is an atom of $\left[S_{i_{j}}, S_{i_{j}+1}\right]$. Set $k:=\sup \left\{i_{j} \mid j \in\right.$ $\left.\mathbb{N}_{m}\right\}, I_{1}:=\left\{j \in \mathbb{N}_{m} \mid i_{j}<k\right\}$ and $I_{2}:=\left\{j \in \mathbb{N}_{m} \mid i_{j}=k\right\}$. In particular, $I_{2} \neq \emptyset$. Then, for each $h \in I_{1}$ and for each $l \in I_{2}$, we have $T_{h} \subseteq S_{k} \subset T_{l} \subseteq S_{k+1}$, so that $U=\left(\prod_{h \in I_{1}} T_{h}\right)\left(\prod_{l \in I_{2}} T_{l}\right)=\prod_{l \in I_{2}} T_{l}$. Then $U \in\left[S_{k}, S_{k+1}\right]$ and property $(\mathcal{P})$ holds.

The following definitions are needed for our study.

Definition 3.26. An integral extension $R \subseteq S$ is called infra-integral [22] (resp.; subintegral [33]) if all its residual extensions $\kappa_{R}(P) \rightarrow \kappa_{S}(Q)$, (with $Q \in \operatorname{Spec}(S)$ and $P:=Q \cap R$ ) are isomorphisms (resp.; and the natural map $\operatorname{Spec}(S) \rightarrow \operatorname{Spec}(R)$ is bijective). An extension $R \subseteq S$ is called t-closed (cf. [22]) if the relations $b \in S, r \in R, b^{2}-r b \in R, b^{3}-r b^{2} \in R$ imply $b \in R$. The $t$-closure ${ }_{S}^{t} R$ of $R$ in $S$ is the smallest element $B \in[R, S]$ such that $B \subseteq S$ is t-closed and the greatest element $B^{\prime} \in[R, S]$ such that $R \subseteq B^{\prime}$ is infra-integral. An extension $R \subseteq S$ is called seminormal (cf. [33]) if the relations $b \in S, b^{2} \in R, b^{3} \in R$ imply $b \in R$. The seminormalization ${ }_{S}^{+} R$ of $R$ in $S$ is the smallest element $B \in[R, S]$ such that $B \subseteq S$ is seminormal and the greatest element $B^{\prime} \in[R, S]$ such that $R \subseteq B^{\prime}$ is subintegral. The canonical decomposition of an arbitrary extension $R \subset S$ is $R \subseteq{ }_{S}^{+} R \subseteq{ }_{S}^{t} R \subseteq \bar{R} \subseteq S$.

Next proposition describes the link between the elements of the canonical decomposition and minimal extensions.

Proposition 3.27. [28, Proposition 4.5] Let there be an integral extension $R \subset S$ and a maximal chain $\mathcal{C}$ of $R$-subextensions of $S$, defined by $R=R_{0} \subset \cdots \subset R_{i} \subset$ $\cdots \subset R_{n}=S$, where each $R_{i} \subset R_{i+1}$ is minimal. The following statements hold:

(1) $R \subset S$ is subintegral if and only if each $R_{i} \subset R_{i+1}$ is ramified.

(2) $R \subset S$ is seminormal and infra-integral if and only if each $R_{i} \subset R_{i+1}$ is decomposed.

(3) $R \subset S$ is t-closed if and only if each $R_{i} \subset R_{i+1}$ is inert.

If either (1) or (2) holds, then $\operatorname{Spec}(S) \rightarrow \operatorname{Spec}(R)$ is bijective.

Example 3.28. We now give three examples of an FCP distributive, hence FIP (and not Boolean) extension where the property $(\mathcal{P})$ holds or not. 
(1) Set $G:=\mathbb{Z} / 12 \mathbb{Z}$, which is a cyclic group, and let $k \subset L$ be a cyclic extension with Galois group $G$. The proper subgroups of $G$ are $2 G, 3 G, 4 G$ and $6 G$, so that the lattice $\mathcal{G}$ of subgroups of $G$ is $\{0,2 G, 3 G, 4 G, 6 G, G\}$, which is distributive [31, Exercise 15, page 125]. Using the reversing order isomorphism of lattices $\psi: \mathcal{G} \rightarrow[k, L]$ defined by $\psi(H):=\operatorname{Fix}(H)$, we obtain the following lattice $[k, L]=\{k, \operatorname{Fix}(i G), L \mid i=2,3,4,6\}$. Set $L_{i}:=\psi(i G)$. We have the following diagram:

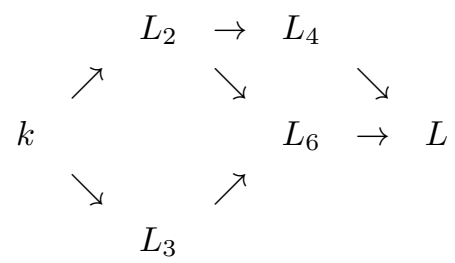

Then, $k \subset L$ is distributive but does not satisfy property $(\mathcal{P})$. Indeed, $L_{6}$ is the socle of $k \subset L$, because $L_{2}$ and $L_{3}$ are the atoms of $k \subset L$, with $L_{6} \subset L$ minimal, so that $S_{0}=k, S_{1}=L_{6}$ and $S_{2}=L$. Moreover, $L_{4} \notin\left[S_{0}, S_{1}\right] \cup\left[S_{1}, S_{2}\right]$. In particular, we cannot apply Corollary 3.24. Indeed, $\mathcal{R}[k, L]=L_{4} \cap L_{6} \neq S_{1}=L_{6}$.

This example shows that the results of Theorem 3.22 hold even if property $(\mathcal{P})$ is not satisfied, since $S_{0} \subset S_{1}$ and $S_{1} \subset S_{2}$ are Boolean. This also shows that in a distributive extension, a $\Pi$-irreducible element is not necessarily an atom of some $\left[S_{j}, S_{j+1}\right]$ (see $L_{4}$ ), and an atom of some $\left[S_{j}, S_{j+1}\right]$ is not necessarily $\Pi$-irreducible (see $L$ ).

(2) An obvious example of a distributive $\mathcal{P}$-extension $R \subset S$ is when $[R, S]$ is a chain.

(3) We give here a more involved example of a distributive $\mathcal{P}$-extension. Take $k \subset L$ a finite separable field extension of degree 6 , such that $[k, L]$ is a Boolean algebra with $[k, L]=\left\{k, L_{1}, L_{2}, L\right\}$ and $\ell[k, L]=2[29$, Example 4.17]. Set $S:=$ $L[X] /\left(X^{2}\right)$, so that $L \subset S$ is a minimal ramified extension and $S$ is a local ring, with maximal ideal $M$. Set $T:={ }_{S}^{t} k$ which is a local ring because $k \subset S$ is a finite integral extension. Then, $T \subset S$ is t-closed with $M=(T: S) \in \operatorname{Max}(T)$ ([10, Lemma 3.17]). Since $k \subset S$ has FCP by [7, Theorem 4.2], there exists a finite chain $k:=R_{0} \subset \cdots \subset R_{n} \subset R_{n+1}:=T$ such that $R_{i} \subset R_{i+1}$ is minimal ramified for each $i=0, \ldots, n$. There are no decomposed minimal extension $R_{i} \subset R_{i+1}$ because $T$ is local. Set $R:=R_{n}$ and consider the extension $R \subset S$. Since $T / M \cong k$ and $S / M \cong L$, we get that $T \subset S$ is a Boolean extension by [29, Proposition 3.5 (5)] with $[T, S]=\left\{T, T_{1}, T_{2}, S\right\}$, where $T_{i}$ is such that $T_{i} / M=L_{i}$ because of the bijection $[T, S] \rightarrow[k, L]$ given by $U \mapsto U / M$. Moreover, $\ell[R, S]=3$ by $[25$, 
Proposition 3.2]. We claim that $T$ is the only atom of the extension. Assume there exists some $T^{\prime} \in[R, S] \backslash\{T\}$ such that $R \subset T^{\prime}$ is minimal. We get that $R \subset T^{\prime}$ can be neither decomposed (as we already observed since $S$ is local) nor ramified, because in this case, we should have $T^{\prime} \subset T$, a contradiction. If $R \subset T^{\prime}$ is minimal inert, this leads also to a contradiction, because $T$ would not be the tclosure, since some minimal ramified extensions would start from $T$ ([8, Proposition 7.4]). Then, $T$ is the socle $S_{1}$ of the extension. Moreover $S=S_{2}$ since $T \subset S$ is Boolean. Consider some $U \in] R, S]$ and let $V \in[R, U]$ be such that $R \subset V$ is minimal. As we already observed, $V=T$ so that $U \in[T, S]=\left[S_{1}, S_{2}\right]$, which yields $[R, S]=\left[S_{0}, S_{1}\right] \cup\left[S_{1}, S_{2}\right]$. We have the following diagram:

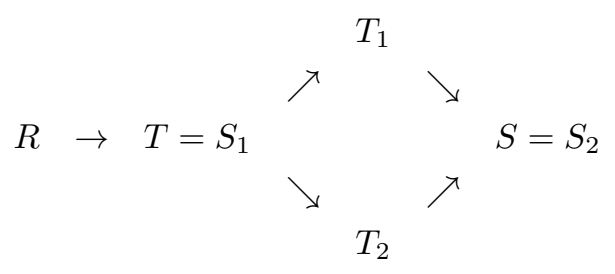

Then, $R \subset S$ is a $\mathcal{P}$-extension and $R \subset S$ is distributive by Corollary 3.22 .

Let $R \subset S$ be a ring extension. We recall that $R$ is called unbranched in $S$ if $\bar{R}$ is local. In this case, if $R \subset S$ has FCP, the following Lemma shows that any $T \in[R, S]$ is local. An extension $R \subset S$ is called quasi-Prüfer if $\bar{R} \subseteq S$ is a Prüfer extension; that is, $\bar{R} \subseteq T$ is a flat epimorphism for each $T \in[\bar{R}, S][27$, Definition 2.1]. An FCP extension is quasi-Prüfer [27, Corollary 3.3] since an FCP integrally closed extension is Prüfer [27, Proposition 1.3]. The reader may also consult [1, Theorem 2].

Lemma 3.29. Let $R \subset S$ be a quasi-Prüfer extension (for example if $R \subset S$ has $F C P)$, such that $R$ is unbranched in $S$. Then, $T$ is local for each $T \in[R, S]$.

Proof. Obvious.

As we saw in Example 3.28 (3), the t-closure of the extension is the socle of the extension. We are going to show that for some distributive extensions $R \subset S$, the t-closure and the integral closure are elements of the Loewy series.

Proposition 3.30. Let $R \subset S$ be an FCP distributive extension (hence FIP) such that $R$ is unbranched in $S$, and $\left\{S_{i}\right\}_{i=0}^{n}$ is its Loewy series. Then, there exist $k, l \in\{0, \ldots, n\}$ such that $\frac{t}{R} R=S_{k}$ and $\bar{R}=S_{l}$. Moreover, $\left[R, \frac{t}{R} R\right]$ and $[\bar{R}, S]$ are chains. 
Proof. Since $\bar{R}$ is a local ring, so are $R$ and $S_{i}$ for any $i \in \mathbb{N}_{n+1}$ by Lemma 3.29 . Let $M$ be the maximal ideal of $R$.

Since $S_{i}$ is local and $S_{i} \subset S_{i+1}$ is Boolean FIP, it follows from [29, Corollary 3.20] that $S_{i} \subset S_{i+1}$ is either integral or Prüfer. Assume that $S_{i} \subset S_{i+1}$ is Prüfer for some $i \in\{0, \ldots, n-2\}$ and let $j>i$ for $j<n$. We claim that $S_{j} \subset S_{j+1}$ is Prüfer. Deny, so that $S_{i} \subset S_{j+1}$ is neither integral, nor Prüfer. Set $S_{i}^{\prime}:=\overline{S_{i}} S_{j+1}$. Then, $S_{i}^{\prime} \neq S_{i}, S_{j+1}$. It follows that there exist a minimal integral extension $S_{i} \subset T$ and a minimal Prüfer extension $S_{i} \subset U$ with $T, U \in\left[S_{i}, S_{j+1}\right], T \subseteq S_{i}^{\prime}, U \subseteq S_{i+1}$. An application of [28, Lemma 1.5]leads to $\mathcal{C}\left(S_{i}, T\right) \neq \mathcal{C}\left(S_{i}, U\right)$, a contradiction since $S_{i}$ is local. Then, $S_{j} \subset S_{j+1}$ is Prüfer for any $j>i$. In particular, as soon as some $S_{k} \subset S_{k+1}$ is Prüfer, so is $S_{i} \subset S_{i+1}$ for each $i \geq k$, whence $S_{k}=\bar{R}$.

Now, if $R \neq \bar{R}$, we can work with the extension $R \subset \bar{R}$, which is also distributive, and its Loewy series is $\left\{S_{i}\right\}_{i=0}^{k}$. From [29, Proposition 3.25], we deduce that $S_{i} \subset$ $S_{i+1}$ is either infra-integral (more precisely subintegral since $S_{i+1}$ is local), or tclosed, for each $i<k$ since $S_{i}$ is local by Proposition 3.27. In order to establish the result for the t-closure, we mimic the previous proof given for the integral closure. Assume that $S_{i} \subset S_{i+1}$ is t-closed and let $j>i$ for $j<k$. We claim that $S_{j} \subset S_{j+1}$ is t-closed. Deny, so that $S_{i} \subset S_{j+1}$ is neither infra-integral, nor t-closed. Set $S_{i}^{\prime}:={ }_{S_{j+1}}^{t} S_{i}$. Then, $S_{i}^{\prime} \neq S_{i}, S_{j+1}$. It follows that there exist a minimal ramified extension $S_{i} \subset T$ and a minimal inert extension $S_{i} \subset U$ with $T, U \in\left[S_{i}, S_{j+1}\right], T \subseteq S_{i}^{\prime}, U \subseteq S_{i+1}$. Then [8, Proposition 7.4 ] shows that there are two maximal chains in $\left[S_{i}, T U\right]$ of different lengths, contradicting the distributivity of $R \subset S$ by Proposition 2.4. Then, $S_{j} \subset S_{j+1}$ is t-closed for any $j$ such that $k>j>i$. In particular, as soon as $S_{l} \subset S_{l+1}$ is t-closed, so is $S_{i} \subset S_{i+1}$ for each $i \geq l$, giving $S_{l}=\frac{t}{R} R$.

Since $\bar{R}$ is a local ring, and $R \subseteq S_{l}$ is subintegral, so is $S_{i} \subset S_{i+1}$ for each $i<l$, and then is minimal ramified by [29, Lemma 3.27]. In particular, each $S_{i} \subset S_{i+1}$ has only one atom, which is $S_{i+1}$. Let $\left.T \in\right] R, S_{l}[$. Since $R \subset T$ has FCP, there is a tower $R \subset T_{i} \subset T$, where $\left\{T_{i}\right\}_{i=0}^{r}$ is the Loewy series of $R \subset T$. Since $R=S_{0}=T_{0}$, an obvious induction shows that $T_{i}=S_{i}$ for all $i<r$, and $T=S_{r}$ for some $r \leq l$, so that $R \subseteq \frac{t}{R} R$ is a chain. By [7, Theorem 6.10], $\bar{R} \subset S$ is a chain.

Remark 3.31. Example 3.16 shows that the conclusion of Proposition 3.30 does not hold in general. In this example, $R$ is not local (although $\operatorname{Spec}(S) \rightarrow \operatorname{Spec}(R)$ is injective) and ${ }_{S}^{t} R=R_{1} \neq S_{i}$ for $i=1,2$ because $R \subset R_{1}$ is minimal ramified and $R_{1} \subset S$ is t-closed. A more precise study will be made in a forthcoming paper. 
When an FCP distributive extension satisfies property $(\mathcal{P})$, the Loewy series allows to give information about the extension.

Proposition 3.32. Let $R \subset S$ be an FCP distributive (hence FIP) $\mathcal{P}$-extension and $\left\{S_{i}\right\}_{i=0}^{n}$ its Loewy series, so that each $S_{i} \subset S_{i+1}$ is Boolean. Then

(1) There exist $j, k \in\{0, \ldots, n\}$ with $j \leq k$ such that $\frac{t}{R} R \in\left[S_{j}, S_{j+1}[\right.$ and $\bar{R} \in\left[S_{k}, S_{k+1}[\right.$.

(2) Let $i \in\{0, \ldots, n-1\}$. If $i<j, S_{i} \subset S_{i+1}$ is infra-integral. If $j<i<$ $k, S_{i} \subset S_{i+1}$ is t-closed. If $i>k, S_{i} \subset S_{i+1}$ is Prüfer.

(3) For $i \in\{0, \ldots, n-1\}$, let $\mathcal{A}_{i}$ be the set of atoms of $\left[S_{i}, S_{i+1}\right]$. Then $\ell[R, S]=$ $\sum_{i=0}^{n-1}\left|\mathcal{A}_{i}\right|$.

Proof. Each $S_{i} \subset S_{i+1}$ is Boolean because of Proposition 3.8.

(1) Since $[R, S]=\cup_{i=0}^{n-1}\left[S_{i}, S_{i+1}\right]$, there exist $j, k \in\{0, \ldots, n\}$ such that $\frac{t}{R} R \in$ $\left[S_{j}, S_{j+1}\left[\right.\right.$ and $\bar{R} \in\left[S_{k}, S_{k+1}\left[\right.\right.$. Moreover, $j \leq k$ since $S_{j} \subseteq \frac{t}{R} R \subseteq \bar{R} \subset S_{k+1}$ implies $j<k+1$.

(2) Let $i<k$ so that $i+1 \leq k$ which implies $S_{i} \subset S_{i+1} \subseteq S_{k} \subset \bar{R}$. Then $S_{i} \subset S_{i+1}$ is integral. If $i<j$, then $S_{i} \subset S_{i+1} \subseteq S_{j} \subseteq \frac{t}{R} R$ and $S_{i} \subset S_{i+1}$ is infraintegral. If $j<i<k$, then $j+1 \leq i$, so that $\frac{t}{R} R \subset S_{j+1} \subseteq S_{i} \subset S_{i+1} \subseteq S_{k} \subseteq \bar{R}$ and $S_{i} \subset S_{i+1}$ is t-closed.

Let $i>k$, so that $i \geq k+1$ which implies $\bar{R} \subset S_{k+1} \subseteq S_{i} \subset S_{i+1}$. Then $S_{i} \subset S_{i+1}$ is Prüfer.

(3) By $[18$, Lemma 4, p.486], we get that $\ell[R, S]$ is the number of $\Pi$-irreducible elements of $] R, S]$. Then, Theorem 3.25 gives the result.

We end this section by studying the Loewy series of some special extensions. We need the following lemma used in the next Proposition.

Lemma 3.33. [24, Lemma 2.9 and the paragraph before Proposition 2.3] An FCP extension $R \subset S$ with a factorization $R \subseteq T \subseteq S$ such that $\operatorname{Supp}_{R}(T / R) \cap$ $\operatorname{Supp}_{R}(S / T)=\emptyset$ admits a unique factorization $R \subseteq U \subseteq S$ such that $T \cap U=R$ and $T U=S$ (U is the complement of $T$ in $[R, S])$. Moreover, $\operatorname{Supp}_{R}(U / R)=$ $\operatorname{Supp}_{R}(S / T)$ and $\operatorname{Supp}_{R}(S / U)=\operatorname{Supp}_{R}(T / R)$.

Proposition 3.34. Let $R \subset S$ be an FCP extension with Loewy series $\left\{S_{i}\right\}_{i=0}^{n}$ and $T \in] R, S\left[\right.$ such that $\operatorname{Supp}_{R}(T / R) \cap \operatorname{Supp}_{R}(S / T)=\emptyset$. Then:

(1) $T$ has a unique complement $U \in[R, S]$.

(2) Let $\left\{T_{i}\right\}_{i=0}^{m}$ (resp.; $\left.\left\{U_{i}\right\}_{i=0}^{r}\right)$ be the Loewy series of $[R, T]$ (resp.; of $[R, U]$ ). Then $S_{i}=T_{i} U_{i}$ for each $i \in\{0, \ldots, n\}$, with $n=£[R, S]=\sup \{m, r\}=$ $\sup \{£[R, T], £[R, U]\}$. 
Proof. (1) Lemma 3.33 gives $T \cap U=R$ and $T U=S(*)$. Moreover $U$ is unique satisfying these properties.

(2) Set $\mathcal{M}:=\operatorname{MSupp}_{R}(S / R), \mathcal{M}_{T}:=\operatorname{MSupp}_{R}(T / R)$ and $\mathcal{M}_{U}:=\operatorname{MSupp}_{R}(U / R)$ $=\operatorname{MSupp}_{R}(S / T)$. Then, $\mathcal{M}=\mathcal{M}_{T} \cup \mathcal{M}_{U}$ with $\mathcal{M}_{T} \cap \mathcal{M}_{U}=\emptyset(* *)$ by Lemma 3.33. Now, we use Proposition 3.14.

Let $M \in \mathcal{M}$ and $\left\{S_{i}^{\prime}\right\}_{i=0}^{n_{M}}$ (resp.; $\left\{T_{i}^{\prime}\right\}_{i=0}^{m_{M}},\left\{U_{i}^{\prime}\right\}_{i=0}^{r_{M}}$ ) be the Loewy series of $\left[R_{M}, S_{M}\right]$ (resp.; $\left.\left[R_{M}, T_{M}\right],\left[R_{M}, U_{M}\right]\right)$. Then $S_{i}^{\prime}=\left(S_{i}\right)_{M}$ for each $i \in\left\{0, \ldots, n_{M}\right\}$, $T_{i}^{\prime}=\left(T_{i}\right)_{M}$ for each $i \in\left\{0, \ldots, m_{M}\right\}$ and $U_{i}^{\prime}=\left(U_{i}\right)_{M}$ for each $i \in\left\{0, \ldots, r_{M}\right\}$. In view of (**), we have either $M \in \mathcal{M}_{T}$ (a), or $M \in \mathcal{M}_{U}$ (b). In case (a), $M \notin \mathcal{M}_{U}$, so that $U_{M}=R_{M}$ and $T_{M}=S_{M}$. It follows that $n_{M}=m_{M}$ and $S_{i}^{\prime}=T_{i}^{\prime}$ for each $i \leq n_{M}$. Moreover, $U_{i}^{\prime}=R_{M}$ for each $i \leq n_{M}$, so that $S_{i}^{\prime}=T_{i}^{\prime} U_{i}^{\prime}=\left(S_{i}\right)_{M}=\left(T_{i}\right)_{M}\left(U_{i}\right)_{M}=\left(T_{i} U_{i}\right)_{M}$. The same reasoning shows that in case (b), $n_{M}=r_{M}$ and $\left(S_{i}\right)_{M}=\left(T_{i}\right)_{M}\left(U_{i}\right)_{M}=\left(T_{i} U_{i}\right)_{M}$, so that $\left(S_{i}\right)_{M}=\left(T_{i} U_{i}\right)_{M}$ for any $M \in \operatorname{MSupp}_{R}(S / R)$. From Corollary 3.15, we deduce that

$$
\begin{aligned}
n & =\sup _{M \in \mathcal{M}}\left(n_{M}\right) \\
& =\sup \left[\sup _{M \in \mathcal{M}_{T}}\left(n_{M}\right), \sup _{M \in \mathcal{M}_{U}}\left(n_{M}\right)\right] \\
& =\sup \left[\sup _{M \in \mathcal{M}_{T}}\left(m_{M}\right), \sup _{M \in \mathcal{M}_{U}}\left(r_{M}\right)\right] \\
& =\sup (m, r) .
\end{aligned}
$$

If $m=r$, then $n=m=r$. Let $i \leq n$. Then, $\left(S_{i}\right)_{M}=\left(T_{i}\right)_{M}\left(U_{i}\right)_{M}$ for each $M \in \mathcal{M}$ leads to $S_{i}=T_{i} U_{i}$.

If $m \neq r$, assume $m<r$, so that $n=r$. As above, $S_{i}=T_{i} U_{i} \in[T, S]$ for each $i \leq m$. Recall that $T_{i}=T$ for each $i \in\{m, \ldots, r\}$. In particular, $S_{m}=$ $T_{m} U_{m}=T U_{m} \in[T, S]$ and we still have $S_{i}=T_{i} U_{i} \in[T, S]$ for each $i \geq m$. Then, $\operatorname{MSupp}_{R}\left(S / S_{i}\right) \subseteq \operatorname{MSupp}_{R}(S / T)=\mathcal{M}_{U}$ for each $i \leq m$.

In [27, Definition 4.1], we call an extension $R \subset S$ almost-Prüfer if it can be factored $R \subseteq U \subseteq S$, where $R \subseteq U$ is Prüfer and $U \subseteq S$ is integral. Actually, $U$ is the Prüfer hull $\widetilde{R}$ of the extension.

Corollary 3.35. Let $R \subset S$ be an FCP almost-Prüfer extension with Loewy series $\left\{S_{i}\right\}_{i=0}^{n}$. Let $\left\{T_{i}\right\}_{i=0}^{m}$ (resp. $\left.\left\{U_{i}\right\}_{i=0}^{r}\right)$ be the Loewy series of $[R, \bar{R}]$ (resp.; of $[R, \widetilde{R}]$ ). Then $S_{i}=T_{i} U_{i}$ for each $i \in\{0, \ldots, n\}$, with $n=£[R, S]=\sup \{£[R, \bar{R}], £[R, \widetilde{R}]\}=$ $\sup \{m, r\}$. 
Proof. We have $\operatorname{Supp}_{R}(\bar{R} / R) \cap \operatorname{Supp}_{R}(S / \bar{R})=\emptyset[27$, Proposition 4.16], with $\widetilde{R}$ the unique $U \in[R, S]$ such that $\bar{R} \cap U=R$ and $\bar{U}=S$. Then, Proposition 3.34 gives the result.

Given a ring $R$, recall that its Nagata ring $R(X)$ is the localization $R(X)=$ $T^{-1} R[X]$ of the ring of polynomials $R[X]$ with respect to the multiplicatively closed subset $T$ of all polynomials with content $R$. In [9, Theorem 32], Dobbs and the authors proved that when $R \subset S$ is an extension, whose Nagara extension $R(X) \subset$ $S(X)$ has FIP, the map $\varphi:[R, S] \rightarrow[R(X), S(X)]$ defined by $\varphi(T)=T(X)$ is an order-isomorphism. We show now that this map send the Loewy series of $R \subset S$ to the Loewy series of $R(X) \subset S(X)$.

Proposition 3.36. Let $R \subset S$ be an extension such that $R(X) \subset S(X)$ has FIP. If $\left\{S_{i}\right\}_{i=0}^{n}$ is the Loewy series of $[R, S]$, then $\left\{S_{i}(X)\right\}_{i=0}^{n}$ is the Loewy series of $[R(X), S(X)]$. In particular, $£[R, S]=£[R(X), S(X)]$.

Proof. Since the map $\varphi:[R, S] \rightarrow[R(X), S(X)]$ defined by $\varphi(T)=T(X)$ is an order-isomorphism, it is also a lattice isomorphism. In particular, if $T, U \in[R, S]$ is such that $T \subset U$ is minimal, so is $T(X) \subset U(X)$ [10, Theorem 3.4]. Let $\left\{S_{i}^{\prime}\right\}_{i=0}^{m}$ be the Loewy series of $[R(X), S(X)]$. Then, an obvious induction on $i$ shows that $S_{i}^{\prime}=S_{i}(X)$ for each $i \in\{0, \ldots, n\}$ since an atom $A$ of $S_{i} \subset S$ gives the atom $A(X)$ of $S_{i}(X) \subset S(X)$, and all atoms of $S_{i}(X) \subset S(X)$ are of this form. In particular, $n=£[R, S]=m=£[R(X), S(X)]$.

Remark 3.37. If $R \subset S$ is an FCP extension such that $R(X) \subset S(X)$ is distributive, then $R(X) \subset S(X)$ has FIP because it has FCP by [10, Theorem 3.9]. In this case, $R \subset S$ is distributive [29, Proposition 3.7], and then has FIP.

In Corollary 3.4 and in Remark 3.5, we proved that for a ring extension $R \subset$ $S$, the two socles $\mathcal{S}[R, S]$ and $\mathcal{M S}[R, S]$ may differ. Using idealization, we may associate to some modules a ring extension. The Loewy length of some modules can be computed as the Loewy length of a ring extension. Let $M$ be an $R$-module. The Loewy length of the $R$-module $M$ is denoted by $\lambda(M)$. We consider the ring extension $R \subseteq R(+) M$, where $R(+) M$ is the idealization of $M$ in $R$.

Recall that $R(+) M:=\{(r, m) \mid(r, m) \in R \times M\}$ is a commutative ring whose operations are defined as follows:

$(r, m)+(s, n)=(r+s, m+n)$ and $(r, m)(s, n)=(r s, r n+s m)$

Then $(1,0)$ is the unit of $R(+) M$, and $R \subseteq R(+) M$ is a ring morphism defining $R(+) M$ as an $R$-module, so that we can identify any $r \in R$ with $(r, 0)$. 
Proposition 3.38. Let $M$ be an $R$-module with finite length and let $S:=R(+) M$ be the idealization of $M$. Let $\left\{S_{i}\right\}_{i=0}^{n}$ be the Loewy series of the ring extension $[R, S]$. Then $S_{i}=R(+) M_{i}$ for each $i \in\{0, \ldots, n\}$ where $\left\{M_{i}\right\}_{i=0}^{n}$ is the Loewy series of the lattice $\Lambda(M)$. In particular, $£[R, S]=\lambda(M)$.

Proof. Since $M$ is an $R$-module with finite length, $R \subseteq R(+) M$ is an FCP quadratic extension [26, Propositions 2.2 and 2.3] and [15, Lemma 2]. Moreover, there is an order isomorphism $\psi: \Lambda(M) \cong[R, R(+) M]$ given by $\psi(N)=R(+) N$. In particular, for $N, N^{\prime} \in \Lambda(M)$ such that $N \subset N^{\prime}$, [26, Proposition 2.8] says that $R(+) N \subset R(+) N^{\prime}$ is minimal if and only if $N^{\prime} / N$ is a simple $R$-module. At last, for $N, N^{\prime} \in \Lambda(M)$, obviously $(R(+) N)\left(R(+) N^{\prime}\right)=R(+)\left(N+N^{\prime}\right)$, so that $\psi$ is also a lattice isomorphism. Let $\left\{S_{i}\right\}_{0}^{m}$ be the Loewy series of $R \subseteq R(+) M$. Then, an easy induction on $i$ shows that $S_{i}=R(+) M_{i}$ for each $i \in\{0, \ldots, n\}$, where $\left\{M_{i}\right\}_{i=0}^{n}$ is the Loewy series of the lattice $\Lambda(M)$. Indeed, an atom $N^{\prime}$ of $M / M_{i}$ is of the form $N / M_{i}$, where $N \in \Lambda(M)$ and $M_{i} \subset N$, and gives the atom $R(+) N$ of $R(+) M_{i} \subset R(+) M$. Moreover, all atoms of $R(+) M_{i} \subset R(+) M$ are of this form. In particular, $£[R, S]=\lambda(M)$.

Example 3.39. Let $R$ be a SPIR (that is a special principal ideal ring with a unique non zero prime ideal $M=R t$ such that $M$ is nilpotent of index $p>0$, a positive integer). Then $R$ is an $R$-module whose set $\Lambda(R)$ of $R$-submodules is the set of ideals $\left\{R, R t^{k} \mid k \in \mathbb{N}_{p}\right\}$. As $\Lambda(R)$ is a chain, we get that $\Lambda(R)=\left\{S_{i}\right\}_{i=0}^{p}$ is the Loewy series of the $R$-module $R$, where $S_{0}=R$, so that $\left\{R(+) S_{i}\right\}_{i=0}^{p}$ is the Loewy series of the ring extension $R \subset R(+) R$, and $[R, R(+) R]$ is a chain by Corollary 3.9.

\section{Finite (distributive) field extension}

We first consider a finite field extension $k \subset L$ with separable closure $T$ and radicial closure $U$. The Loewy series of $k \subset L$ is linked to those of $k \subset T$ and $k \subset U$. If $k \subset L$ is a radicial extension, then $\mathrm{c}(k)$ is a prime number. We recall that a minimal field extension is either radicial, or separable [23, Remark before Proposition 2.2, page 371]. If $K$ is an atom of $[k, L]$, then $k \subset K$ is either radicial or separable. In the first case, we say that $K$ is a radicial atom, and in this case, $[K: k]=p=\mathrm{c}(k)$. In the second case, $K$ is a separable atom. If $k \subset L$ is a finite field extension which is not separable, let $T$ be its separable closure, so that $T \subset L$ is a radicial extension. In particular, $p:=\mathrm{c}(T)$ is a prime number, so that $c(k)=p$. Since a finite dimensional separable field extension has FIP, we consider 
in this section mainly FIP field extension. We found less results for FCP not FIP field extensions. We begin with the following lemma.

Lemma 4.1. Let $k \subset L$ be an FCP field extension with separable closure $T$, radicial closure $U$ such that $T, U \notin\{k, L\}$. Let $T^{\prime} \in[k, T]$ and $U^{\prime} \in[k, U]$. Set $K:=T^{\prime} U^{\prime}$. Then:

(1) $U^{\prime} \subset K$ is separable and $T^{\prime} \subset K$ is radicial.

(2) If there exists $U^{\prime \prime} \in[k, U]$ such that $U^{\prime \prime}$ is a radicial atom of $\left[U^{\prime}, U\right]$, then $K U^{\prime \prime}$ is a radicial atom of $[K, L]$. Moreover, if $k \subset L$ has FIP, then $K U^{\prime \prime}$ is the only radicial atom of $[K, L]$.

(3) The separable atoms $V$ of $[K, L]$ are of the form $V=K T^{\prime \prime}$, where $T^{\prime \prime}$ is an atom of $T^{\prime} \subset T$. In particular, $T^{\prime \prime}=V \cap T$.

Proof. Since $k \subset U$ is radicial, $p:=\mathrm{c}(k)$ is a prime number.

(1) Obvious, because $T^{\prime}$ (resp.: $U^{\prime}$ ) is generated by a separable element (resp.; radicial elements) over $k$, which implies that this element generates a separable (resp.; these elements generate a radicial) extension $U^{\prime} \subset K=U^{\prime} T^{\prime}$ (resp.; $T^{\prime} \subset$ $\left.K=U^{\prime} T^{\prime}\right)$.

(2) For the same reason, $K \subset K U^{\prime \prime}$ is radicial, and of degree $p$, since $\left[U^{\prime \prime}\right.$ : $\left.U^{\prime}\right]=p$. In particular, $K U^{\prime \prime}$ is a radicial atom of $K \subset L$. Moreover, assume that $k \subset L$ has FIP. Then, $K U^{\prime \prime}$ is the unique radicial atom of $[K, L]$. Deny and let $W \in[K, L], W \neq K U^{\prime \prime}$, be a radicial atom of $[K, L]$. Then $K \subset U^{\prime \prime} W$ is a finite radicial extension which is not a chain, a contradiction. It follows that $K U^{\prime \prime}$ is the only radicial atom of $[K, L]$.

(3) Let $V$ be a separable atom of $[K, L]$. We have the following diagram,

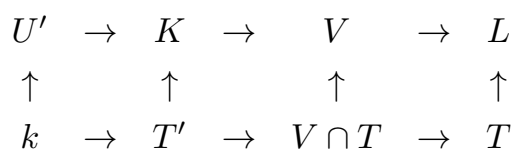

where $V \cap T$ is the separable closure of $T^{\prime} \subset V$. In particular, $V \cap T \subseteq V$ is radicial, with $V \cap T \neq T^{\prime}$ because $T^{\prime} \subset V$ is not radicial, and $V \cap T \neq V$ because $T^{\prime} \subset V$ is not separable. We claim that $V$ is of the form $K T^{\prime \prime}$, where $T^{\prime \prime}$ is an atom of $T^{\prime} \subset T$. Since $\left.\left.V \cap T \in\right] T^{\prime}, T\right]$, there is some $\left.\left.T^{\prime \prime} \in\right] T^{\prime}, V \cap T\right]$ such that $T^{\prime} \subset T^{\prime \prime}$ is minimal. Then, $T^{\prime} \subset T^{\prime \prime} \subseteq V \cap T$ implies $K=U^{\prime} T^{\prime} \subseteq U^{\prime} T^{\prime \prime} \subseteq U^{\prime}(V \cap T) \subseteq V$. But $K \subset V$ minimal implies that either $U^{\prime} T^{\prime \prime}=K(*)$ or $U^{\prime} T^{\prime \prime}=V(* *)$. In case (*), we have $T^{\prime \prime} \in\left[T^{\prime}, K\right]$, with $T^{\prime} \subset K$ radicial, so that $T^{\prime} \subset T^{\prime \prime}$ is both radicial and separable, a contradiction. So, only case (**) holds and $V=U^{\prime} T^{\prime \prime}=U^{\prime}(V \cap T)$ $=U^{\prime} T^{\prime} T^{\prime \prime}=K T^{\prime \prime}$, because $T^{\prime} \subset T^{\prime \prime}$. In particular, $T^{\prime \prime}=V \cap T$, as the separable closure of $T^{\prime} \subset V$ because $T^{\prime \prime} \subseteq U^{\prime} T^{\prime \prime}=V$ is radicial. 
Conversely, an atom $T^{\prime \prime}$ of $\left[T^{\prime}, T\right]$ is such that $T^{\prime} \subset T^{\prime \prime}$ is minimal separable. The inclusion $T^{\prime} \subset T^{\prime \prime}$ leads to $K=U^{\prime} T^{\prime} \subseteq U^{\prime} T^{\prime \prime}$ separable, with $T^{\prime \prime} \subset U^{\prime} T^{\prime \prime}$ radicial and $K \neq U^{\prime} T^{\prime \prime}$ by a similar reasoning as before. In particular, $T^{\prime \prime}=U^{\prime} T^{\prime \prime} \cap T$ as the separable closure of $T^{\prime} \subseteq U^{\prime} T^{\prime \prime}$. Assume that $K \subset U^{\prime} T^{\prime \prime}$ is not minimal, so that there exists $V \in] K, U^{\prime} T^{\prime \prime}$, with $K \subset V$ separable. Then, $T^{\prime}=K \cap T \subseteq$ $V \cap T \subseteq U^{\prime} T^{\prime \prime} \cap T=T^{\prime \prime}$. As above, $V \cap T \neq T^{\prime}$, because $T^{\prime} \subseteq V$ is not radicial, which leads to $V \cap T=T^{\prime \prime}$, and then to $U^{\prime} T^{\prime \prime}=U^{\prime}(V \cap T) \subseteq V$, a contradiction. Then, $K \subset U^{\prime} T^{\prime \prime}$ is minimal.

Definition 4.2. [12] A finite field extension $k \subset L$ is said to be exceptional if $k=L_{r}$ and $L_{s} \neq L$.

Proposition 4.3. Let $k \subset L$ be an FIP field extension with separable closure $T$, radicial closure $U$ and $T, U \notin\{k, L\}$. Let $\left\{S_{i}\right\}_{i=0}^{n}$ (resp. $\left.\left\{T_{i}\right\}_{i=0}^{m},\left\{U_{i}\right\}_{i=0}^{r},\left\{T_{i}\right\}_{i=m}^{s}\right)$ be the Loewy series of $k \subset L$ (resp. $k \subset T, k \subset U, T \subset L)$. Then:

(1) If $i \leq \inf (m, r)$, then $S_{i}=T_{i} U_{i}$.

(2) If $m \leq r$, then $S_{i}=T_{i+m}$ for $i \geq m$. In particular, $£[k, L]=n=s-m$.

(3) If $r<m$ and $U \subset L$ is separable, then $U$ is the complement of $T$. Moreover, $S_{i}=U_{r} T_{i}=U T_{i}$ for $i \in\{r, \ldots, m\}$, with $L=S_{n}=T_{s}=U T_{n}$. In particular, $£[k, L]=n=m=£[k, T]$ and $s=£[k, T]+£[k, U]=m+r$.

(4) Assume that $r<m$ and $U \subset L$ is not separable. For $i \geq r$, the $S_{i}$ 's are gotten by induction on $i$ in the following way: if $S_{i} \subset L$ is exceptional, then $S_{i+1}=S_{i} T_{i+1}$; if $S_{i} \subset L$ is not exceptional, then $S_{i+1}=V_{i} T_{i+1}$, where $V_{i}$ is the unique radicial atom of $\left[S_{i}, L\right]$. In this way, we obtain the family $\left\{S_{i}\right\}_{i=r}^{t}$, for the least $t$ such that $S_{t} \in\left[T_{m}, L\right]$. As there exists some l such that $S_{t}=T_{l}$, then $S_{i}=T_{l+i-t}$, for $i \geq t$. In particular, $n=s+t-l$.

Proof. Set $p:=\mathrm{c}(k)$.

(1) We show by induction on $i$ that $S_{i}=T_{i} U_{i}$ for any $i \leq \inf (m, r)$. For $i=0$, we have $k=S_{0}=T_{0}=U_{0}=T_{0} U_{0}$. Assume that for some $i<\inf (m, r)$, we have $S_{i}=T_{i} U_{i}$. We are going to determine the atoms of $S_{i} \subset L$. Since any minimal field extension is either radicial or separable, it is enough to characterize any $V \in\left[S_{i}, L\right]$ which is either a radicial atom $(*)$, or a separable atom $(* *)$. We use Lemma 4.1. In case $(*), V=S_{i} U_{i+1}$, since $U_{i+1}$ is the only radicial atom of $\left[U_{i}, L\right]$. In case (**), $V=S_{i} T^{\prime \prime}$, where $T^{\prime \prime}$ is any atom of $T_{i} \subset T$. Because $S_{i+1}$ is the product of all atoms of $S_{i} \subset L$, we get that $S_{i+1}=S_{i} U_{i+1} T_{i+1}$. But, $S_{i}=T_{i} U_{i}$ with $T_{i} \subset T_{i+1}$ and $U_{i} \subset U_{i+1}$ leads to $S_{i+1}=U_{i+1} T_{i+1}$, and the induction is proved.

(2) Assume that $m \leq r$. In view of (1), we have $S_{m}=T_{m} U_{m}=T U_{m}$ with $S_{m} \subseteq L$ radicial. Indeed, $T_{m} \subset L$ is radicial, and then a chain, and $S_{m} \in\left[T_{m}, L\right]=$ 
$\left\{T_{i}\right\}_{i=m}^{s}$. We have the following diagram

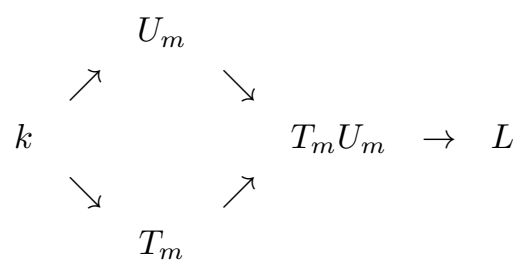

with $k \subset U_{m}, T_{m} \subset T_{m} U_{m}$ both radicial, and $k \subset T_{m}, U_{m} \subset T_{m} U_{m}$ both separable. In particular, $\left[U_{m}: k\right]=\left[T_{m} U_{m}: T_{m}\right]=p^{m}$ since $U_{i+1}$ is a radicial atom of $\left[U_{i}, L\right]$ for each $i \in\{0, \ldots, m-1\}$. It follows that $T_{m} U_{m}=S_{m}=T_{2 m}$ and $S_{i}=T_{m+i}$ for any $i \in\{m, \ldots, n\}$, so that $m+n=s$ because $S=S_{n}=T_{s}=T_{m+n}$.

(3) Assume that $m>r$, so that $S_{r}=T_{r} U_{r}=U T_{r}$. Let $V$ be an atom of $S_{r} \subset L$. If $V$ is a separable atom of $\left[S_{r}, L\right]$, then $V=S_{r} T^{\prime \prime}$, where $T^{\prime \prime}$ is an atom of $T_{r} \subset T$ in view of Lemma 4.1. In particular, $S_{r} T_{r+1}=U_{r} T_{r} T_{r+1}=U_{r} T_{r+1}$ is the product of the separable atoms of $S_{r} \subset L$. Since $U \subset L$ is separable, there is no radicial atom in $\left[S_{r}, L\right]$. It follows that $S_{r+1}=U_{r} T_{r+1}$. An obvious induction shows that $S_{i}=U_{r} T_{i}$ for any $i \in\{r, m\}$. Since $T U \in[T, L] \cap[U, L]$, we get $L=T U$. Therefore, $U$ is a complement of $T$ because $k=T \cap U$, which is obviously unique. Moreover, $T=T_{m} \subset U_{r} T_{m}$ and $U=U_{r} \subset U_{r} T_{m}$, so that $L=U_{r} T_{m}=S_{m}=S_{n}=T_{s}=U T_{n}$ since $U \subset L$ is separable and $T \subset L$ is radicial. Then, $n=m$. Moreover, $s=m+r$ since $[L: T]=[U: k]=p^{r}=p^{s-m}$.

(4) Assume that $r<m$ and that $U \subset L$ is not separable. We have $S_{r}=U_{r} T_{r}$ by (1). We get by induction on $i \geq r$ the $S_{i}$ 's in the following way: Assume that $S_{i}$ is gotten. If $S_{i} \subset L$ is exceptional, there is no $V \in\left[S_{i}, L\right]$ such that $S_{i} \subset V$ is minimal radicial, then $S_{i+1}=S_{i} T_{i+1}$ as in case (3). If $S_{i} \subset L$ is not exceptional, there is a unique radicial atom $V_{i}$ of $\left[S_{i}, L\right]$. But in this case, since $S_{i} T_{i+1}$ is the product of separable atoms of $\left[S_{i}, L\right]$, then $S_{i+1}=S_{i} T_{i+1} V_{i}=V_{i} T_{i+1}$ because $S_{i} \subset V_{i}$. Since $\left\{S_{i}\right\}_{i=r}^{n}$ is an increasing sequence, there is a least $t \geq r$ such that $S_{t} \in\left[T_{m}, L\right]$. Indeed, $T_{m} \subseteq S_{m}$, and there exists some $l \geq m$ such that $S_{t}=T_{l}$ because $\left[T_{m}, L\right]=\left\{T_{i}\right\}_{i=m}^{s}$. Then $S_{i}=T_{l+i-t}$, for $i \geq t$, which implies that $s=l+n-t$, that is $n=s+t-l$.

Proposition 4.4. Let $k \subset L$ be an FCP radicial field extension. Set $p:=\mathrm{c}(k)$ and $[L: k]=p^{n}$.

(1) Then $\mathcal{S}[k, L]=\left\{x \in L \mid x^{p} \in k\right\}$.

(2) If $k \subset L$ has FIP, the Loewy series of $k \subset L$ is $[k, L]$ and $£[k, L]=n$.

Proof. (1) $\mathcal{S}[k, L]=\prod_{A \in \mathcal{A}} A$, where $\mathcal{A}$ is the set of atoms of $[k, L]$. Now, $A \in$ $\mathcal{A} \Leftrightarrow k \subset A$ is minimal $\Leftrightarrow A=k[x]$ with $k \subset k[x]$ minimal radicial $\Leftrightarrow[k[x]$ : 
$k]=p \Leftrightarrow A=k[x]$ with $x^{p} \in k$. In particular, $t^{p} \in k$ for any $t \in A$ since $\mathrm{c}(k)=p$. Let $y \in \mathcal{S}[k, L]$. Then $y$ is a finite sum of products $z:=x_{1} \cdots x_{n}$ of elements of atoms of $[k, L]$. But $z^{p}=x_{1}^{p} \cdots x_{n}^{p} \in k$, which yields that $y^{p} \in k$. Then, $\mathcal{S}[k, L] \subseteq\left\{x \in L \mid x^{p} \in k\right\}$. Conversely, if $x^{p} \in k$ for some $x \in L \backslash k$, it follows that $k \subset k[x]$ is minimal, so that $k[x] \in \mathcal{A}$ which leads to $x \in \mathcal{S}[k, L]$. Then, $\mathcal{S}[k, L]=\left\{x \in L \mid x^{p} \in k\right\}$.

(2) Obvious since $[k, L]$ is a chain.

Remark 4.5. Contrary to FCP separable field extensions which are always FIP, there exist FCP radicial field extensions which are not FIP. Take for instance $k:=$ $\mathbb{Z} / 2 \mathbb{Z}(Y, T)$, the field of rational functions over $\mathbb{Z} / 2 \mathbb{Z}$ in two indeterminates $Y$ and $T$. Let $\alpha$ (resp. $\beta$ ) be a zero of $F(X):=X^{2}-Y\left(\operatorname{resp} . G(X):=X^{2}-T\right)$ in the same algebraically closed field extension of $k$. Then, $k \subset k[\alpha, \beta]$ is an FCP radicial extension of length 2. But, it has not FIP by [28, Theorem 6.1 (8) (a)], because $|[k, k[\alpha, \beta]]| \geq 4$ gives $|[k, k[\alpha, \beta]]|=\infty$.

Actually, the following proposition gives a characterization of FIP radicial field extensions.

Proposition 4.6. Let $k \subset L$ be an FCP radicial field extension. The following conditions are equivalent:

(1) $k \subset L$ is a chain.

(2) $k \subset L$ is distributive.

(3) $k \subset L$ has FIP.

Proof. $(1) \Rightarrow(2)$ by [29, Proposition 2.3].

(1) $\Rightarrow(3)$ since $k \subset L$ has FCP.

$(3) \Rightarrow(1)$ by $[29$, Lemma 4.1].

(2) $\Rightarrow(1)$ Assume that $k \subset L$ is distributive and not a chain. There exist $K_{1}, K_{2}, K_{3} \in[k, L]$ such that $K_{1} \subset K_{i}$ is minimal for $i=2,3$ with $K_{2} \neq K_{3}$. We get that $\left|\left[K_{1}, K_{2} K_{3}\right]\right|=4$ because $\ell\left[K_{1}, K_{2} K_{3}\right] \mid=2$. Indeed, $k \subset L$ is distributive, so that $\left[K_{1}, K_{2} K_{3}\right]$ does not contain a diamond [13, Theorem 1, page 59], a contradiction with [28, Theorem 6.1 (8) (a)].

The last case to consider is the case of a finite separable field extension. We recall here some results gotten in [29].

Let $L:=k[x]$ be a finite separable (whence FIP) field extension of $k$ and $f(X) \in$ $k_{u}[X]$ (the set of monic polynomials of $k[X]$ ) the minimal polynomial of $x$ over $k$. If $g(X) \in L_{u}[X]$ divides $f(X)$, we denote by $K_{g}$ the $k$-subalgebra of $L$ generated by the coefficients of $g$. For any $K \in[k, L]$, we denote by $f_{K}(X) \in K_{u}[X]$ the 
minimal polynomial of $x$ over $K$. The proof of the Primitive Element Theorem shows that $K=K_{f_{K}}$. Of course, $f_{K}(X)$ divides $f(X)$ in $K[X]$ (and in $L[X]$ ). We set $\mathcal{D}:=\left\{f_{K} \mid K \in[k, L]\right\}$. Then, $(\mathcal{D}, \leq)$ is a poset for the order $\leq$ defined as follows: if $f_{K}, f_{K^{\prime}} \in \mathcal{D}$, then $f_{K} \leq f_{K^{\prime}}$ if and only if $f_{K}$ divides $f_{K^{\prime}}$ in $L[X]$, which is equivalent to $K^{\prime} \subseteq K$ by [29, Lemma 4.7]. In particular, inf is gcd in $\mathcal{D}$.

Corollary 4.7. [29, Corollary 4.9] The map $\varphi:[k, L] \rightarrow \mathcal{D}$ defined by $K \mapsto f_{K}$ is a reversing order bijection such that $f_{K K^{\prime}}=\inf \left(f_{K}, f_{K^{\prime}}\right)$ for $K, K^{\prime} \in[k, L]$.

Proposition 4.8. Let $k \subset L:=k[x]$ be a finite separable field extension of $k$ and $K \subset K^{\prime}$ a subextension. Then, $K \subset K^{\prime}$ is minimal if and only if $f_{K^{\prime}}$ is a maximal proper divisor of $f_{K}$ in $\mathcal{D}$.

Proof. In view of Corollary 4.7, we have $K \subset K^{\prime}$ if and only if $f_{K^{\prime}}$ divides $f_{K}$ in $\mathcal{D}$. Moreover, $K \subset K^{\prime}$ is minimal if and only if there is no $K^{\prime \prime} \in[k, L]$ such that $K \subset K^{\prime \prime} \subset K^{\prime}$ if and only if there is no proper divisor of $f_{K}$ divided strictly by $f_{K^{\prime}}$ if and only if $f_{K^{\prime}}$ is a maximal proper divisor of $f_{K}$ in $\mathcal{D}$.

Proposition 4.9. Let $k \subset L:=k[x]$ be a finite separable field extension of $k$. The Loewy series $\left\{S_{i}\right\}_{i=0}^{n}$ of $k \subset L$ is gotten by induction in the following way: $S_{0}=k$ and for $i \in\{0, \ldots, n-1\}$, we have $S_{i+1}=K_{g}$, where $g=\inf \{h \in \mathcal{D} \mid h$ is a maximal proper divisor of $f_{S_{i}}$ in $\left.\mathcal{D}\right\}$.

Proof. For a given $S_{i}$, we have $S_{i+1}=\prod\left\{V \mid S_{i} \subset V\right.$ minimal $\}$. In view of Proposition 4.8, $S_{i} \subset V$ is minimal $\Leftrightarrow f_{V}$ is a maximal proper divisor of $f_{S_{i}}$. It follows from Corollary 4.7 that $S_{i+1}=K_{g} \Leftrightarrow g=f_{S_{i+1}}=\inf \{h \in \mathcal{D} \mid h$ is a maximal proper divisor of $\left.f_{S_{i}}\right\}$.

Example 4.10. We have seen in Example 3.28 (1) how we can get the Loewy series of a finite cyclic field extension $k \subset L:=k[x]$ of degree 12. Assume that $k$ contains a primitive 12 th root of unity and that $x^{12}=a \in k$. According to [19, Proposition 9.6 and Corollary 9.7], the minimal polynomial of $x$ over $k$ is $P_{1}(T)=T^{12}-a$. Moreover, any element of $[k, L]$ is of the form $k[y]$, where $y=x^{j}$ for some divisor $j$ of 12 . Set $i:=12 / j$, so that $y^{i}=a$. Then, keeping the notation of Example 3.28 (1) and Proposition 4.9, we get $L_{i}=k\left[x^{j}\right]$ and the minimal polynomial of $x$ over $L_{i}$ is $P_{i}(T)=T^{j}-x^{j} \in L_{i}[X]$ (of degree $j=12 / i$ ), so that $\mathcal{D}=\left\{P_{i}(T) \mid i=1,2,3,4,6,12\right\}$. Since $S_{0}=k$ and $P_{1}(T)=T^{12}-a$, the maximal proper divisors of $f_{S_{0}}=P_{1}$ are $P_{2}$ and $P_{3}$, with $P_{6}=\inf \left\{P_{2}, P_{3}\right\}$. Then, we recover $S_{1}=L_{6}$ as in Example 3.28(1). Now, $S_{2}=L$ is obvious. 
Proposition 4.11. Let $k \subset L:=k[x]$ be a minimal separable field extension. Let $N$ be the normal closure of $L$. Then, $£[k, N]=1$. Moreover, the following conditions are equivalent:

(1) $k \subset N$ is distributive.

(2) $k \subset N$ is Boolean.

(3) $k \subset N$ is a cyclic extension and $[N: k]$ is square-free.

Proof. Let $G$ be the Galois group of $k \subset N$. Then $N=k[\{\sigma(x) \mid \sigma \in G\}]=$ $\prod_{\sigma \in G} k[\sigma(x)]$. Since $k \subset k[\sigma(x)]$ is obviously minimal for any $\sigma \in G$, it follows that $N=\mathcal{S}[k, N]$, so that $£[k, N]=1$.

$(1) \Leftrightarrow(2)$ by Lemma 3.6 and by definition of a Boolean extension.

$(2) \Leftrightarrow(3)$ by [29, Theorem 4.19].

Corollary 4.12. Let $k \subset L$ be a finite Galois extension with Galois group $G$. The socle $\mathcal{S}[k, L]$ is globally invariant by the elements of $G$. Moreover, $k \subset \mathcal{S}[k, L]$ is Galois.

Proof. By definition, $\mathcal{S}[k, L]=\prod_{A \in \mathcal{A}} A$. For any $A \in \mathcal{A}$, there exists $x_{A} \in A$ such that $A=k\left[x_{A}\right]$, so that $\mathcal{S}[k, L]=\prod_{A \in \mathcal{A}} k\left[x_{A}\right]$. For any $\sigma \in G$, we have $\sigma(\mathcal{S}[k, L])=\sigma\left(\prod_{A \in \mathcal{A}} k\left[x_{A}\right]\right)=\prod_{A \in \mathcal{A}} k\left[\sigma\left(x_{A}\right)\right]$. Obviously, $k\left[\sigma\left(x_{A}\right)\right] \in \mathcal{A}$ which yields $\sigma(\mathcal{S}[k, L]) \subseteq \mathcal{S}[k, L]$. But $\sigma$ being a $k$-isomorphism, for any $x \in L$ such that $k[x] \in \mathcal{A}$, and setting $y:=\sigma^{-1}(x)$, that is $x=\sigma(y)$, we have $k[x]=k[\sigma(y)]$, with $k[y] \in \mathcal{A}$. To conclude, $\mathcal{S}[k, L] \subseteq \sigma(\mathcal{S}[k, L])$ and $\mathcal{S}[k, L]=\sigma(\mathcal{S}[k, L])$. This equality shows that $k \subset \mathcal{S}[k, L]$ is Galois by [3, Proposition 5, page A V.54].

We give here a complete study of the case of finite Galois distributive field extensions. They are evidently FIP. Recall that a finite Galois field extension is minimal if and only if its degree is a prime integer [23, Proposition 2.2] and is Boolean if and only if it is a cyclic extension with a square-free degree [29, Theorem 4.19].

Proposition 4.13. A finite Galois field extension is distributive if and only if it is cyclic.

Proof. Let $k \subset L$ be a finite Galois field extension with Galois group $G$ and let $\mathcal{G}$ be the set of subgroups of $G$. In view of the Fundamental Theorem of Galois Theory, the maps $\varphi:[k, L] \rightarrow \mathcal{G}$ defined by $\varphi(K):=\operatorname{Aut}_{K}(L)$, the group of $K$-automorphisms of $L$, for each $K \in[k, L]$ and $\psi: \mathcal{G} \rightarrow[k, L]$ defined by $\psi(H):=\operatorname{Fix}(H)$, the fixed field of $H$ in $L$, for each $H \in \mathcal{G}$ are reversing order isomorphisms of lattices, with $\varphi=\psi^{-1}$ (see [3, Corollaire 2, page A V.65]). It follows 
that $k \subset L$ is distributive if and only if $\mathcal{G}$ is distributive if and only if $G$ is cyclic [14, Theorem 19.2.1].

Theorem 4.14. Let $k \subset L$ be a finite cyclic field extension. Set $r:=[L: k]=$ $\prod_{i=1}^{m} p_{i}^{\alpha_{i}}$, where the $p_{i}$ are distinct prime integers. Let $\mathbb{D}_{r}$ be the set of divisors of $r$ and $\mathcal{A}$ be the set of atoms of $[k, L]$. Then:

(1) For each $i \in \mathbb{N}_{m}$, there is a unique $A_{i} \in \mathcal{A}$ such that $\left[A_{i}: k\right]=p_{i}$. Let $T \in[k, L]$. Then, $T \in \mathcal{A}$ if and only if $[T: k]=p_{i}$ for some $i \in \mathbb{N}_{m}$.

(2) $\mathcal{S}[k, L]=\prod_{i=1}^{m} A_{i}$.

(3) Set $\alpha:=\sup \left\{\alpha_{i} \mid i \in \mathbb{N}_{m}\right\}$. Let $\left\{S_{j}\right\}_{j=0}^{n}$ be the Loewy series of $k \subset L$ and let $j \in\{0, \ldots, n\}$. Then, $\left[S_{j}: k\right]=\prod_{i=1}^{m} p_{i}^{\beta_{i}}$, where either $\beta_{i}=\alpha_{i}$ if $\alpha_{i}<j$ or $\beta_{i}=j$ if $\alpha_{i} \geq j$. Moreover, $T \in[k, L]$ is an atom of $\left[S_{j}, S_{j+1}\right]$ if and only if $[T: k]=\prod_{i=1}^{m} p_{i}^{\gamma_{i}}$, where there exists a unique $i_{0}$ such that $\beta_{i_{0}}<\alpha_{i_{0}}$, satisfying $\gamma_{i}=\beta_{i}$ for each $i \neq i_{0}$ and $\gamma_{i_{0}}=\beta_{i_{0}}+1$. In particular, $\alpha=n=£[k, L]$.

(4) $S_{j} \subset S_{j+1}$ is Boolean for each $j \in\{0, \ldots, n-1\}$.

(5) Let $T \in] k, L]$. Then, $T$ is a $\Pi$-irreducible element of $[k, L]$ if and only if $[T: k]=p_{i}^{\beta_{i}}$ for some $\beta_{i} \in \mathbb{N}_{\alpha_{i}}$ and some $i \in \mathbb{N}_{m}$.

(6) $|[k, L]|=\mathrm{d}([L: k])=\prod_{i=1}^{m}\left(\alpha_{i}+1\right)=\left|\mathbb{D}_{r}\right|$. Let $\mathcal{A}_{j}$ be the set of atoms of $\left[S_{j}, S_{j+1}\right]$. Then, $\ell[k, L]=\sum_{j=0}^{\alpha-1}\left|\mathcal{A}_{j}\right|=\sum_{i=1}^{m} \alpha_{i}$ which is the number of $\Pi$-irreducible elements of $] k, L]$.

(7) The following conditions are equivalent:

(a) $k \subset L$ is a $\mathcal{P}$-extension.

(b) $k \subset L$ is either Boolean or a chain.

(c) either $m=1$ or $\alpha=1$.

Proof. First observe that any subextension of $k \subset L$ is still cyclic and $k \subset L$ is Galois distributive. Moreover, there is a lattice isomorphism $\mathbb{D}_{r} \rightarrow[k, L]$, where $d \mapsto T$ such that $[T: k]=d$.

(1) Let $G$ be the Galois group of $k \subset L$. Then, $G \cong \mathbb{Z} / r \mathbb{Z}$, with $|G|=r$. Since $k \subset L$ is cyclic, it follows that for any integer $d \in \mathbb{D}_{r}$, there exists a unique $T \in[k, L]$ such that $[T: k]=d$, and conversely, $[T: k] \in \mathbb{D}_{r}$ for any $T \in[k, L]$. In particular, $k \subset T$ is minimal if and only if $[T: k]$ is a prime integer. Then, $T \in \mathcal{A}$ if and only if $[T: k]=p_{i}$ for some $i \in \mathbb{N}_{m}$, and for each $i \in \mathbb{N}_{m}$, there is a unique $A_{i} \in \mathcal{A}$ such that $\left[A_{i}: k\right]=p_{i}$.

(2) By definition, $\mathcal{S}[k, L]=\prod_{A \in \mathcal{A}} A=\prod_{i=1}^{m} A_{i}$.

(3) We show by induction on $j \geq 0$ that $\left[S_{j}: k\right]=\prod_{i=1}^{m} p_{i}^{\beta_{i}}$, where either $\beta_{i}=\alpha_{i}$ if $\alpha_{i}<j$ or $\beta_{i}=j$ if $\alpha_{i} \geq j$. The induction hypothesis holds clearly for $j=0$. 
Assume that the induction hypothesis holds for some $j \in\{0, \ldots, n-1\}$ so that $S_{j} \subset L$. Then, $\left[S_{j}: k\right]=\prod_{i=1}^{m} p_{i}^{\beta_{i}}$, where either $\beta_{i}=\alpha_{i}$ if $\alpha_{i}<j$ or $\beta_{i}=j$ if $\alpha_{i} \geq j$. Set $m_{j}:=\left|\left\{\alpha_{i} \in \mathbb{N}_{m} \mid \alpha_{i}>j\right\}\right|$. There is no harm to renumber the $\alpha_{i}$ 's so that $\alpha_{i}>j$ for each $i \leq m_{j}$ and $\alpha_{i} \leq j$ for each $i>m_{j}$. Let $T$ be an atom of $\left[S_{j}, S_{j+1}\right]$, so that $S_{j} \subset T$ is minimal and $\left[T: S_{j}\right]=p_{i_{0}}$, for some $i_{0} \in \mathbb{N}_{m}$. In particular, $[T: k]=p_{i_{0}}^{\beta_{i_{0}}+1} \prod_{i \neq i_{0}} p_{i}^{\beta_{i}}$, so that $\beta_{i_{0}}+1 \leq \alpha_{i_{0}}$, which leads to $\beta_{i_{0}}<\alpha_{i_{0}}$. Then, $\beta_{i_{0}}=j$, that is $\alpha_{i_{0}}>j$ and $i_{0} \leq m_{j}$.

Conversely, if $i \leq m_{j}$, then $\alpha_{i}>j$, so that there exists $T \in\left[S_{j}, L\right]$ such that $\left[T: S_{j}\right]=p_{i}$, and $T$ is an atom of $S_{j} \subset L$.

Since $S_{j+1}$ is the product of all atoms of $S_{j} \subset L$, it follows that $\left[S_{j+1}: S_{j}\right]=$ $\prod_{i=1}^{m_{j}} p_{i}$, giving

$$
\left[S_{j+1}: k\right]=\left[S_{j+1}: S_{j}\right]\left[S_{j}: k\right]=\left(\prod_{i=1}^{m_{j}} p_{i}\right)\left(\prod_{i=m_{j}+1}^{m} p_{i}^{\beta_{i}}\right)\left(\prod_{i=1}^{m_{j}} p_{i}^{\beta_{i}}\right)=\prod_{i=1}^{m} p_{i}^{\beta_{i}^{\prime}},
$$

where $\beta_{i}^{\prime}=\beta_{i}+1$ if $i \leq m_{j}$ and $\beta_{i}^{\prime}=\beta_{i}$ for $i>m_{j}$. This means the following: if $\alpha_{i} \geq j+1>j$, then, $\beta_{i}^{\prime}=\beta_{i}+1=j+1$, if $\alpha_{i}<j$, then, $\beta_{i}^{\prime}=\beta_{i}=\alpha_{i}$, and, if $\alpha_{i}=j$, then, $\beta_{i}^{\prime}=\beta_{i}=j=\alpha_{i}$. Hence, the induction hypothesis holds for $S_{j+1}$. Therefore, $L=S_{\alpha}=S_{n}$ and $\alpha=n=£[k, L]$.

(4) $S_{j} \subset S_{j+1}$ is Boolean for each $j \in\{0, \ldots, n-1\}$ by Proposition 3.8 since $k \subset L$ is distributive. In particular, we recover the fact that $\left[S_{j+1}: S_{j}\right]$ is square-free for each $j \in\{0, \ldots, n-1\}$.

(5) Let $T \in] k, L]$ be such that $[T: k]=p_{i}^{\beta_{i}}$ for some $\beta_{i} \in \mathbb{N}_{\alpha_{i}}$ and some $i \in \mathbb{N}_{m}$. It follows that $[k, T]$ is a chain, since so is the Galois group of $k \subset T$ (isomorphic to $\left.\mathbb{Z} / p_{i}^{\beta_{i}} \mathbb{Z}\right)$. Then, $T$ is $\Pi$-irreducible by Proposition 2.5 .

Assume now that $[T: k]$ is divided by at least two distinct prime integers. After a suitable reordering, we may assume that $[T: k]=\prod_{i=1}^{r} p_{i}^{\beta_{i}}, r>1$ and $\beta_{i}>0$ for each $i \in \mathbb{N}_{r}$. In view of the Fundamental Theorem of Galois Theory, there exist $T_{1}, T_{2} \in[k, T]$ such that $\left[T: T_{i}\right]=p_{i}$ for $i=1,2$, so that $T_{1} \subset T$ and $T_{2} \subset T$ are two minimal field extensions as it is recalled before Proposition 4.13. Then, $T$ is not П-irreducible by Proposition 2.5.

(6) The equality $|[k, L]|=\mathrm{d}([L: k])$ is obvious because of the lattice isomorphism recalled at the beginning of the proof. By [18, Lemma 4, p.486], $\ell[k, L]$ is the number of $\Pi$-irreducible elements of $] k, L]$. Then, $\ell[k, L]=\sum_{i=1}^{m} \alpha_{i}$ by (5). To end, $\ell[k, L]=\sum_{j=0}^{\alpha-1} \ell\left[S_{j}, S_{j+1}\right]$ by Proposition 3.8. But each $\left[S_{j}, S_{j+1}\right]$ is Boolean, so that $\ell\left[S_{j}, S_{j+1}\right]=\left|\mathcal{A}_{j}\right|$ again by [18, Lemma 4, page 486] since an element of the Boolean lattice $\left[S_{j}, S_{j+1}\right]$ is a $\Pi$-irreducible element different from $S_{j}$ if and only if it is an atom of $\left[S_{j}, S_{j+1}\right]$. This yields $\ell[k, L]=\sum_{j=0}^{\alpha-1}\left|\mathcal{A}_{j}\right|$. 
We can remark that we may consider $\ell[k, L]$ in two different ways : when we write

$$
\begin{array}{rcccccc}
\ell[k, L]= & 1 & + & \ldots & + & \ldots & =\alpha_{1} \\
1 & + & \ldots & + & \ldots & =\alpha_{2} \\
\ldots & \ldots & \ldots & \ldots & \ldots & \ldots \\
1 & + & \ldots & + & \ldots & =\alpha_{m} \\
= & & = & & & \\
\left|\mathcal{A}_{0}\right| & & \left|\mathcal{A}_{1}\right| & & \ldots &
\end{array}
$$

in line $i$, we have the power of $p_{i}$ in $[L: k]$, which is the number of $\Pi$-irreducible elements whose degree of extension over $k$ is a power of $p_{i}$, and in column $j$, we have the number of atoms of $S_{j} \subset S_{j+1}$, with either 1 or 0 instead of $\ldots$, and 0 after and under each 0 .

(7) We discuss with respect to $m$ and $\alpha$.

If $m=1$, then $k \subset L$ is a chain and a $\mathcal{P}$-extension by Corollary 3.9.

Assume $m>1$.

If $\alpha=1$, then $[L: k]=\prod_{i=1}^{m} p_{i}$ shows that $k \subset L$ is Boolean by the remark before Proposition 4.13, because its degree is square-free, and then a $\mathcal{P}$-extension since $[k, L]=\left[k, S_{1}\right]$.

Assume that $\alpha>1$. After reordering, we may assume that $\alpha_{1}>1$. There exists $T \in[k, L]$ such that $[T: k]=p_{1}^{2}$. Since $\left[S_{1}: k\right]=\prod_{i=1}^{m} p_{i}$ by (3), we get that $T \notin\left[k, S_{1}\right] \cup\left[S_{1} ; L\right]$, so that $[k, L] \neq \cup_{i=0}^{n-1}\left[S_{i}, S_{i+1}\right]$ and $k \subset L$ is not a $\mathcal{P}$-extension in this case. In particular, $k \subset L$ is neither Boolean nor a chain.

Gathering the different cases we get (7).

Example 4.15. Example 3.28 (1) illustrates Theorem 4.14. Since $[L: k]=r=$ $12=3.2^{2}$, we recover results of Theorem 4.14 with $p_{1}=3$ and $p_{2}=2, \alpha_{1}=1$ and $\alpha_{2}=2$, giving that $S_{1}=L_{2} L_{3}=L_{6}$, the product of the atoms of $k \subset L$, and $S_{2}=$ $L$, because $L_{6} \subset L$ is minimal. Moreover, $|[k, L]|=6=\mathrm{d}(12), \ell[k, L]=3$, where the $\Pi$-irreducible elements of $] k, L]$ are $L_{2}, L_{3}$ and $L_{4}$, and $£[R, S]=\sup \left\{\alpha_{1}, \alpha_{2}\right\}=$ 2 .

Remark 4.16. Comparing Theorem 3.22 and Theorem 4.14 (4), we see that the latter is a generalization of Theorem 3.22 in case of a finite cyclic field extension. Indeed, $k \subset L$ is distributive and $S_{j} \subset S_{j+1}$ is Boolean for each $j \in\{0, \ldots, n-1\}$. The condition $\mathcal{P}$ does not matter.

\section{References}

[1] M. Ben Nasr and N. Jarboui, New results about normal pairs of rings with zero divisors, Ric. Mat., 63(1) (2014), 149-155. 
[2] N. Bourbaki; Algèbre Commutative, Chs. 1-2, Hermann, Paris, 1961.

[3] N. Bourbaki, Algèbre, Chs. 4-7, Masson, Paris, 1981.

[4] P.-J. Cahen, G. Picavet and M. Picavet-L'Hermitte, Pointwise minimal extensions, Arab. J. Math. (Springer), 7(4) (2018), 249-271.

[5] G. Călugăreanu, Lattice Concepts of Module Theory, Kluwer Academic Publishers, Dordrecht, 2000.

[6] D. E. Dobbs, B. Mullins, G. Picavet and M. Picavet-L'Hermitte, On the FIP property for extensions of commutative rings, Comm. Algebra, 33(9) (2005), 3091-3119.

[7] D. E. Dobbs, G. Picavet and M. Picavet-L'Hermitte, Characterizing the ring extensions that satisfy FIP or FCP, J. Algebra, 371 (2012), 391-429.

[8] D. E. Dobbs, G. Picavet, M. Picavet-L'Hermitte and J. Shapiro, On intersections and composites of minimal ring extensions, JP J. Algebra Number Theory Appl., 26 (2012), 103-158.

[9] D. E. Dobbs, G. Picavet and M. Picavet-L'Hermitte, When an extension of Nagata rings has only finitely many intermediate rings, each of those is a Nagata ring, Int. J. Math. Math. Sci, (2014), 315919 (13 pp).

[10] D. E. Dobbs, G. Picavet and M. Picavet-L'Hermitte, Transfer results for the FIP and FCP properties of ring extensions, Comm. Algebra, 43 (2015), 12791316.

[11] D. Ferrand and J.-P. Olivier, Homomorphismes minimaux d'anneaux, J. Algebra, 16 (1970), 461-471.

[12] R. Gilmer and W. Heinzer, On the existence of exceptional field extensions, Bull. Amer. Math. Soc., 74 (1968), 545-547.

[13] G. Grätzer, General Lattice Theory, Academic Press, New York-London, 1978.

[14] M. Hall, The Theory of Groups, The Macmillan Co., New York, 1959.

[15] J. A. Huckaba and I. J. Papick, A note on a class of extensions, Rend. Circ. Mat. Palermo (2), 38 (1989), 430-436.

[16] N. Jarboui, A note on the (FMC) condition for extensions of commutative rings, Int. J. Open Problems Comput. Math., 5(3) (2012), 88-95.

[17] M. Knebusch and D. Zhang, Manis Valuations and Prüfer Extensions I, Springer-Verlag, Berlin, 2002.

[18] S. Mac Lane and G. Birkhoff, Algebra, Amer. Math. Soc., 1999.

[19] P. Morandi, Field and Galois Theory, Springer-Verlag, New York, 1996.

[20] C. Năstăsescu and F. Van Oystaeyen, Dimensions of Ring Theory, Mathematics and its applications, D. Reidel Publishing Co., Dordrecht, 1987. 
[21] D. G. Northcott, Lessons on Rings, Modules and Multiplicities, Cambridge University Press, London, 1968.

[22] G. Picavet and M. Picavet-L'Hermitte, T-Closedness, in: Non- Noetherian Commutative Ring Theory, Math. Appl. 520, Kluwer, Dordrecht, (2000), 369386.

[23] G. Picavet and M. Picavet-L'Hermitte, About minimal morphisms, in: Multiplicative Ideal Theory in Commutative Algebra, Springer, New York, (2006), 369-386.

[24] G. Picavet and M. Picavet-L'Hermitte, Prüfer and Morita hulls of FCP extensions, Comm. Algebra, 43 (2015), 102-119.

[25] G. Picavet and M. Picavet-L'Hermitte, Some more combinatorics results on Nagata extensions, Palest. J. Math., 5 (2016), 49-62.

[26] G. Picavet and M. Picavet-L'Hermitte, Modules with finitely submodules, Int. Electron. J. Algebra, 19 (2016), 119-131.

[27] G. Picavet and M. Picavet-L'Hermitte, Quasi-Prüfer extensions of rings, in: Rings, Polynomials and Modules, Springer, (2017), 307-336.

[28] G. Picavet and M. Picavet-L'Hermitte, Rings extensions of length two, J. Algebra Appl., 18(8) (2019), 1950174 (34 pp).

[29] G. Picavet and M. Picavet-L'Hermitte, Boolean FIP ring extensions, Comm. Algebra, 48 (2020), 1821-1852.

[30] G. Picavet and M. Picavet-L'Hermitte, Catenarian FCP ring extensions, to appear in J. Commut. Algebra.

[31] S. Roman, Lattices and Ordered Sets, Springer, New York, 2008.

[32] R. P. Stanley, Enumerative Combinatorics, Vol. 1, Second edition, Cambridge University Press, Cambridge, 2012.

[33] R. G. Swan, On seminormality, J. Algebra, 67 (1980), 210-229.

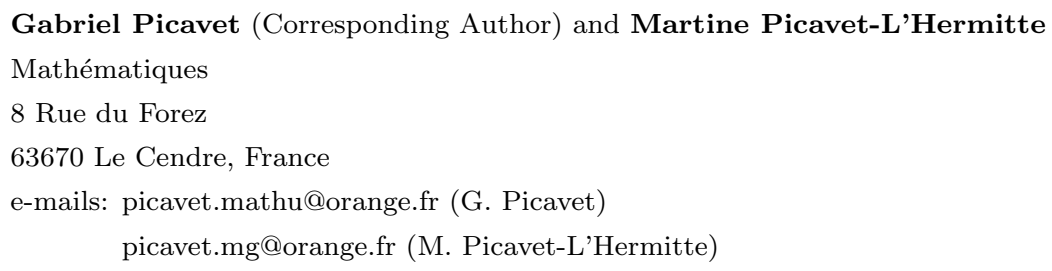

